\title{
Low Temperature Graphitization of Interphase Polyacrylonitrile (PAN)
}

Yiying Zhang, Navid Tajaddod, Kenan Song and Marilyn L. Minus*

Northeastern University, Department of Mechanical and Industrial Engineering, College of Engineering, 360 Huntington Avenue, 334 Snell Engineering Center, Boston, MA 02115-5000.

*Corresponding author. Tel: 617 373-2608. E-mail: m.minus@ neu.edu (Marilyn L. Minus)

\begin{abstract}
Ordered polyacrylonitrile (PAN) interphase structures were formed in solution-cast PAN/carbon nanotube (CNT) composite films by enhancing polymer crystallization conditions and processing parameters for five types of CNTs. All film samples were heat-treated using similar stabilization and carbonization (up to $1100{ }^{\circ} \mathrm{C}$ ) processes. Both the precursor and carbonized materials were characterized by electron microscopy and X-ray spectroscopy. Highly ordered graphitic structure was formed predominantly in the carbonized materials at $1100{ }^{\circ} \mathrm{C}$ (i.e., $\sim 1500{ }^{\circ} \mathrm{C}$ lower than the temperature used in a commercial graphitization process). The ordering of the graphite structure formed at $1100{ }^{\circ} \mathrm{C}$ was further improved by heat treatment up to $2100{ }^{\circ} \mathrm{C}$. Multiple characterization results indicate that the early onset of PAN conversion to graphite is directly related to the polymer interphase formation as well as the CNT type. Based on the stabilization and carbonization parameters used in this study, PAN/single-wall carbon nanotube (SWNT) samples showed more prevalent graphite formation at $1100{ }^{\circ} \mathrm{C}$. This work demonstrates the influence of CNT type regarding interfacial confinement toward this low-temperature polymerto-graphite conversion process.
\end{abstract}




\section{INTRODUCTION}

Presently polyacrylonitrile (PAN) remains the major precursor material for industrial carbon fiber $(\mathrm{CF})$ production due to its relatively high mechanical properties and carbon yield [1-7]. The lightweight and high-performance properties of $\mathrm{CF}$ materials have revolutionized several applications requiring higher energy efficiency (e.g., lightweight and low density composites). However, the production cost for CFs has limited its usage to certain industrial sectors (e.g., transportation and construction). In addition to high cost, the properties of CFs (in particular tensile strength) have remained only a fraction of the theoretical predictions. These limitations continue to drive scientific research related to CF production toward decreasing fabrication costs, improving specimen properties, and increasing fundamental understanding of matrix development during the precursor-to-CF conversion processes for enhancing the fiber structure.

For carbon fiber production, PAN copolymers rather than homopolymers, are typically used, as the structural inhomogeneity along the chain can increase fiber drawability [1, 8-9] and shorten the stabilization time $[1,4,8-10]$. In more recent work, the addition of carbon nanotube (CNT) fillers in the PAN matrix has been shown to contribute to the structural evolution during thermal treatment of the PAN/CNT composite fibers, for potential next-generation CF production [11-12]. The presence of CNTs has been found to reduce both entropic and reaction shrinkages in the matrix and improve the maximum tension that a fiber can endure during stabilization in both oxidative and inert environments [13-14]. Stabilization is a crucial step for the entire heat treatment process toward CF conversion [5, 9, 15-19]. PAN stabilization is a complicated process, which involves structural evolution and chemical reactions including cyclization, oxidation, dehydrogenation, and cross-linking [20-22]. During this process, the presence of 
CNTs can promote the conversion of PAN into a highly ordered ladder structure, which exhibits increased orientation, fewer chain terminations, and longer conjugated segments at the interphase (i.e., near vicinity of CNTs), as compared to the structure conversion of the matrix farther away from the CNTs $[12,14]$. In addition, the presence of CNT-fillers can reduce the formation of $\beta$ amino nitrile groups (which tend to cause chain scission), improves the orientation of the ladder polymer, and increases the tensile modulus of the stabilized fiber [14].

Within polymer-based nano-composites, CNTs and other nano-carbons have shown ability to induce polymer interphase formation [23-30]. In addition to the structural significance of the interphase-polymer (i-polymer) to the precursor composite materials [31-34], more importantly, previous work [11-12, 35-37] has reported that in the near vicinity of the CNTs the PAN conversion to graphite can occur when fibers are only carbonized up to $1100{ }^{\circ} \mathrm{C}$. This phenomenon was initially recognized by Raman spectroscopy characterization of the fibers, which showed intensity increases corresponding to the G-band peak for PAN/single-wall carbon nanotube (SWNT) fibers with $1 \mathrm{wt} \%$ loading [12]. However, a broad carbonaceous peak was also observed at $\sim 25.5^{\circ}$ from X-ray spectroscopy analysis [12] indicating that the fibers consisted predominantly of the turbostratic carbon phase (typically formed at such low carbonization temperatures). High-resolution transmission electron microscopy (HR-TEM) analysis showed that the formation of graphite in these fibers was limited to the local interfacial regions between the polymer matrix and SWNT. This previous work motivates the current study in this paper.

This current work first demonstrates controlled formation of the interphase-PAN ( $i$-PAN) in PAN/CNT films. In other recent work by the authors, studies regarding precise control over the formation of the $i$-PAN structure through coupling epitaxial crystallization [30] and the temperature-dependent PAN crystal growth [38], for the specific PAN copolymer are performed. 
In this foundational paper, various $i$-PAN crystalline coating morphologies on CNTs were well characterized by SEM, TEM and WAXD [30]. In particular, the formation of highly crystalline tubular interfacial coatings of PAN were desired due to their extended-chain structure [30], which may be most compatible with the conversion of precursor $i$-PAN to graphite at lowtemperature [12]. Here, the formation and growth of this extended-chain structure (i.e., $i$-PAN) [30] was also further refined in the current work to produce composite films with predominant $i$ PAN structure (i.e., bulk-free composite films). Second this paper shows that the structure and conformational stability of the $i$-PAN directly affects the early onset of carbonization/graphitization.

In this study, poly(acrylonitrile-co-methacrylic acid) (PAN-co-MAA) random copolymer (for simplicity referred as PAN) and five types of CNT materials were used to fabricate $i$-PAN/CNT composite films. As mentioned, in these film structures the interphase regions dominate in comparison to the bulk polymer matrix in the composites. The solution processing and film fabrication conditions were tailored to achieve the most favorable crystalline interphase growth. A heat treatment procedure similar to what was reported in previous work [12] was adapted here for both the stabilization and carbonization processes. This study provides in-depth insight toward the importance of PAN interphase structure formation for the early onset of graphite in CNT-based materials, which may have significant implications in terms of CF processing.

\section{EXPERIMENTAL SECTION}

\subsection{Materials}


The PAN $\left(M_{w} \sim 513,000 \mathrm{~g} / \mathrm{mol}\right)$ used in this work is a poly(acrylonitrile-co-methacrylic acid) random copolymer with methacrylic acid content of $4 \mathrm{wt} \%$, obtained from Exlan Co. Japan. The tacticity of this PAN polymer was also confirmed experimentally by nuclear magnetic resonance (NMR) spectroscopy [38]. Five types of CNT materials were used: S-A, S-B, F-A, F-B and MIX (see Table 1). Dimethylacetamide (DMAc) (CAS\# 127-19-5) solvent was purchased from Fisher Scientific. All materials were used as-received without further modification.

Table 1. The CNT materials used for PAN composite fabrication.

\begin{tabular}{|c|c|c|c|c|c|c|}
\hline \multicolumn{2}{|c|}{ Material } & S-A & S-B & F-A & F-B & MIX \\
\hline \multirow{3}{*}{\multicolumn{2}{|c|}{$\begin{array}{r}\text { Type } \\
\text { Metallic Impurity } \\
\text { (provided by manufacturer) } \\
\text { Carbonaceous Impurity } \\
\text { (based on EM observations) }\end{array}$}} & SWNT & SWNT & FWNT $^{*}$ & FWNT $^{*}$ & mixture $^{\dagger}$ \\
\hline & & $\sim 94.5 \%$ & $\sim 98.9 \%$ & $93.2 \%$ & $94.8 \%$ & $99 \%$ \\
\hline & & None & None & DWNT $^{*}$ & DWNT $^{*}$ & $\begin{array}{l}\text { onions, } \\
\text { amorphous } \\
\text { carbon, etc. }\end{array}$ \\
\hline \multirow{2}{*}{$\begin{array}{r}\text { Diameter } \\
(\mathrm{nm})\end{array}$} & manufacturer & $0.8-1.2$ & $0.8-1.2$ & - & - & $1-4$ \\
\hline & EM observations & - & - & $5-10$ & $5-10$ & - \\
\hline \multirow{2}{*}{$\begin{array}{r}\text { Length } \\
(\mu \mathrm{m})\end{array}$} & manufacturer & $\sim 0.1-1$ & $\sim 0.1-1$ & $10-30$ & $10-30$ & $3-30$ \\
\hline & EM observations & - & - & - & - & - \\
\hline \multicolumn{2}{|r|}{ Manufacturer } & \multicolumn{4}{|c|}{ Continental Carbon Nanotechnologies, Inc. } & Cheaptubes, Inc. \\
\hline \multicolumn{2}{|c|}{$I_{G / D}$ by Raman spectroscopy } & 11.3 & 7.9 & 37.4 & 32.2 & 16.7 \\
\hline
\end{tabular}

\subsection{Solution Processing and Film Fabrication}

$45 \mathrm{mg}$ of PAN powder was dissolved in $180 \mathrm{~mL}$ DMAc at $90{ }^{\circ} \mathrm{C}$ to form a solution at a concentration of $250 \mathrm{mg} / \mathrm{L} .15 \mathrm{mg}$ of CNTs was subsequently added to this solution and the CNT/PAN/DMAc mixture was transferred immediately into a $90{ }^{\circ} \mathrm{C}$ water bath for $24 \mathrm{hr}$ sonication. Bath sonication was conducted using Fisher F530 bath sonicator (frequency $43 \mathrm{kHz}$, power $150 \mathrm{~W}$ ). After sonication the solution was kept constant at $90{ }^{\circ} \mathrm{C}$ and subjected to shear to 
promote extended-chain PAN crystallization. A negative pressure was used to accelerate solvent evaporation until the solution volume decreased from $180 \mathrm{~mL}$ to $30 \mathrm{~mL}$. The solution temperature for the reduced volume was gradually decreased to $60^{\circ} \mathrm{C}$ until equilibrium. Shearing remained continuous throughout the entire process. The solution was poured into polytetraflouroethylene (PTFE) dish and cast-dried in vacuum oven at $70{ }^{\circ} \mathrm{C}$ for $12 \mathrm{hrs}$. Neat CNT buckypapers/films were fabricated using similar solution processing steps without the addition of PAN polymer.

\subsection{Heat Treatment Processes}

A Lindberg/Blue $\mathrm{M}^{\mathrm{TM}}$ Mini-Mite ${ }^{\mathrm{TM}}$ tube furnace equipped with a quartz tube (diameter 1 inch, obtained from Quartz Scientific Inc.) is used to conduct both stabilization and carbonization procedures. Two flow meters were used to control the inlet and outlet gas flow through the furnace. The entire system was sealed and the outlet gas flow was directly released into a venting system. In this work, the stabilization and carbonization parameters are kept consistent for all samples. The film samples were held in compression using a customized sample holder throughout the heat treatment processes. For stabilization (in air), the temperature was (i) ramped up at $1{ }^{\circ} \mathrm{C} / \mathrm{min}$ from room temperature $\left(25^{\circ} \mathrm{C}\right)$ to $285^{\circ} \mathrm{C}$, (ii) maintained isothermal for $10 \mathrm{hrs}$, (iii) ramped up at $1{ }^{\circ} \mathrm{C} / \mathrm{min}$ from 285 to $330{ }^{\circ} \mathrm{C}$, (iv) maintained isothermal for $3 \mathrm{hrs}$, and (v) gradually decreased to room temperature. For carbonization (in argon), the temperature was (i) ramped up at $5{ }^{\circ} \mathrm{C} / \mathrm{min}$ from room temperature to $1100{ }^{\circ} \mathrm{C}$, (ii) maintained isothermal for $5 \mathrm{~min}$, and (iii) gradually decreased to room temperature $\left(\sim 25^{\circ} \mathrm{C}\right)$. Figure 1 summarizes the timetemperature profile for both the stabilization and carbonization procedures. In both processes, the required air or argon gas flow was maintained constant at $3000 \mathrm{ccm}$ until the chamber is cooled 
down to room temperature $\left(\sim 25^{\circ} \mathrm{C}\right)$. To examine the structural features of the early onset graphite (i.e., formed at $1100{ }^{\circ} \mathrm{C}$ ), the pyrolyzed PAN/S-A composite film was further heattreated at up to $2100{ }^{\circ} \mathrm{C}$ for $40 \mathrm{~min}$ in an argon gas environment.

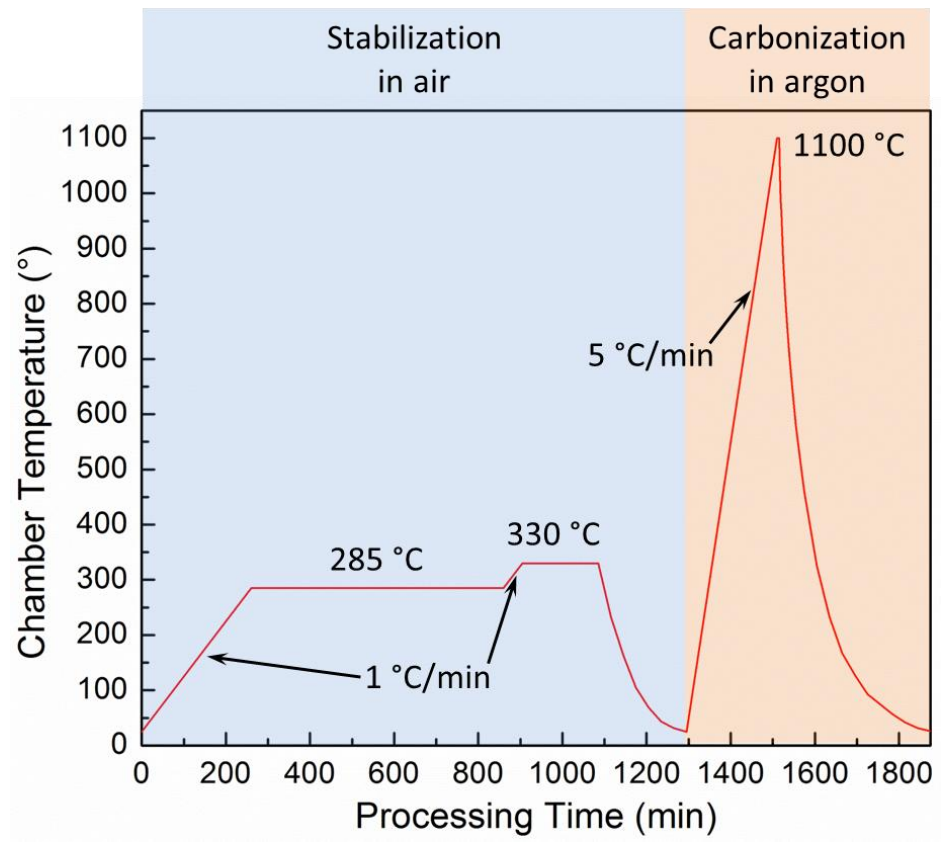

Figure 1. Time-temperature profile for the stabilization and carbonization procedures.

\subsection{Sample Characterization}

Morphology characterization was performed on a Zeiss Supra 25 field emission scanning electron microscope (operating voltage $5 \mathrm{kV}$ ). All film samples were fractured and mounted on a $90^{\circ}$ pin stub with the fractured end facing up for scanning electron microscopy (SEM) observation. Precursor samples were coated with a thin gold/palladium layer $(15-20 \mathrm{~nm})$ for imaging using a Gatan high resolution ion beam coater. High-resolution bright-field transmission electron microscopy (TEM) images were taken using a JEOL 2010 Advance High Performance 
transmission electron microscope (operating voltage: $200 \mathrm{kV}$ ). TEM samples were prepared with the aid of a loop tool (Electron Microscopy Sciences, Cat\# 70944) to place samples directly onto lacey carbon coated copper grids (Electron Microscopy Sciences, Cat\# LC200-Cu). Lattice spacing measurements from TEM images were calibrated using a graphitized carbon black standard (Electron Microscopy Sciences, Cat\# 80037).

Wide-angle X-ray diffraction (WAXD) was performed on a Rigaku RAPID II equipped with a curved detector X-ray diffraction (XRD) system with a $3 \mathrm{~kW}$ sealed tube X-ray source (operating voltage $40 \mathrm{kV}$ and current $30 \mathrm{~mA}$ ). XRD curve fitting and analysis was performed using softwares PDXL 2 (version 2.0.3.0) and 2DP (version 1.0.3.4). Raman spectroscopy was conducted on a Jobin Yvon LabRam HR800 (laser wavelength $532 \mathrm{~nm}$ ). Thermogravimetric analysis (TGA) was performed to investigate the material thermal stability, using the thermogravimetric analyzer (Q50, manufactured by TA Instruments) for the CNT buckypapers and carbonized PAN/CNT composite films with a heating rate of $10{ }^{\circ} \mathrm{C} / \mathrm{min}$ in air from $\sim 25^{\circ} \mathrm{C}$ to $900{ }^{\circ} \mathrm{C}$ and held isothermal for 30 minutes.

\section{RESULTS AND DISCUSSIONS}

In this work, graphitization has been successfully performed at a carbonization temperature of $1100{ }^{\circ} \mathrm{C}$ for specifically designed $i$-PAN/CNT composite precursor films. Films were produced using five CNT types, and carbonized samples incorporating SWNT (i.e., S-A and S-B) exhibit pronounced graphite (002) WAXD peaks. The evolution of the carbonized morphology, structure, and thermal stability for these materials as a function of the CNTs present in the composite is discussed in the subsequent sections. 


\section{1. $i$-PAN Formation on CNTs}

It is well-known that polymer crystallization can be achieved by undercooling a polymer melt or solution. Concentrating polymer solutions via solvent evaporation is also used to aid crystal growth. During these processes, molecules are attracted to each other and pack into long-range three-dimensional order to form a solid mass [39]. In this work, along with a temperature control the solvent was continuously removed from the solution during the crystallization process.

The introduction of shear flow into nano-filler/polymer composite solutions has been shown to create a synergistic effect for promoting directed crystallization, due to the changes in the local stress levels and orientation of chains surrounding the nanoparticles upon the application of shear [40-42]. The synergy between CNT-induced epitaxy and shear-induced chain extension can be understood, where (1) the graphitic structure of CNTs and its unique one-dimensional geometry can induce polymer epitaxy for nucleation at the interfacial region, and (2) the presence of shear flow has an impact on initiating the coil-stretch transition and promoting the subsequent growth of the fibrillar nuclei consisting of extended-chains. Therefore, a shearing step was adapted during the crystallization process in this study.

As a result of refining the crystallization procedure, $i$-PAN coating morphology was found to form on various CNT materials. Representative SEM images show the formation of this structure in SWNT (Figure 2a), FWNT (Figure 2b) and the MIX (Figure 2c) systems, respectively. By using this same processing procedure and crystallization conditions for the PAN/CNT composite system films, a similar $i$-PAN coating was formed in all materials. 

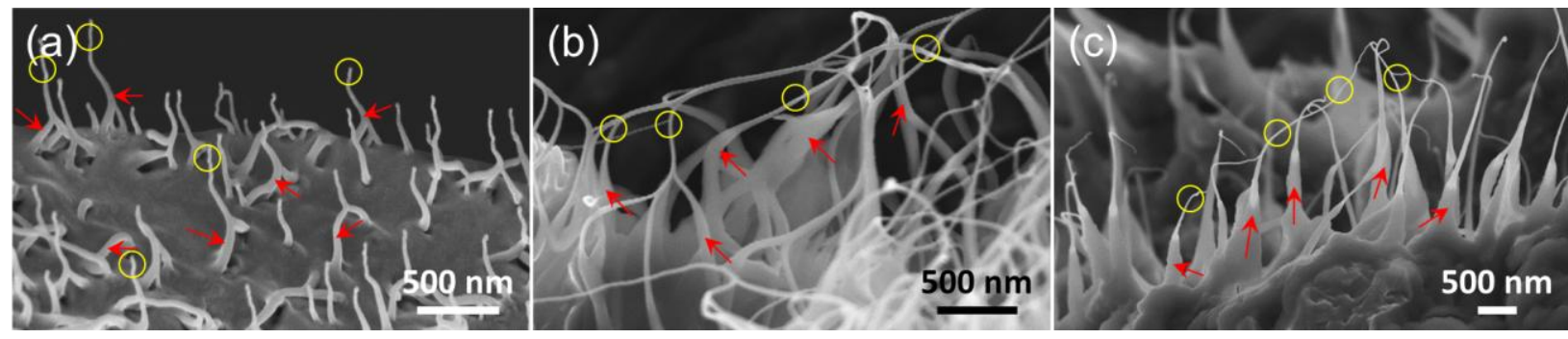

Figure 2. SEM images showing $i$-PAN coating structure on different types of CNTs, including (a) S-A (SWNT), (b) F-A (mixture of DWNT and FWNT), and (c) MIX (mixture of SWNT, DWNT, FWNT and carbonaceous species) in $i$-PAN/CNT composites. Circles indicate CNTs, and arrows indicate $i$-PAN coating.

\subsection{Structure and Morphology of Precursor $i$-PAN/CNT Films}

WAXD spectra for $i$-PAN/CNT composite and PAN control films are provided in Figure 3a. All composite films show a major PAN peak that exhibits a (110)/(200) doublet (Figure 3b). Typically a single (110) peak is observed, as is the case for the PAN control film. The presence of the $(110) /(200)$ doublet is indicative of higher crystal perfection for the PAN polymer in the composites. The peak position (i.e., Bragg angle), corresponding $d$-spacing, and crystal size are listed for each PAN crystal peak in Table 2. All the composites exhibit similar crystal size for the major PAN doublet peak. However, the control PAN film shows a much smaller crystal size. In addition to PAN peaks, a broad carbonaceous peak centered at $\sim 26^{\circ}$ is shown for composite samples incorporating F-A, F-B and MIX fillers (arrows in Figure 3a). This is due to the presence of FWNT and/or layered carbonaceous species (i.e., onion structures), as well as amorphous carbon present in the as-received CNT materials. 


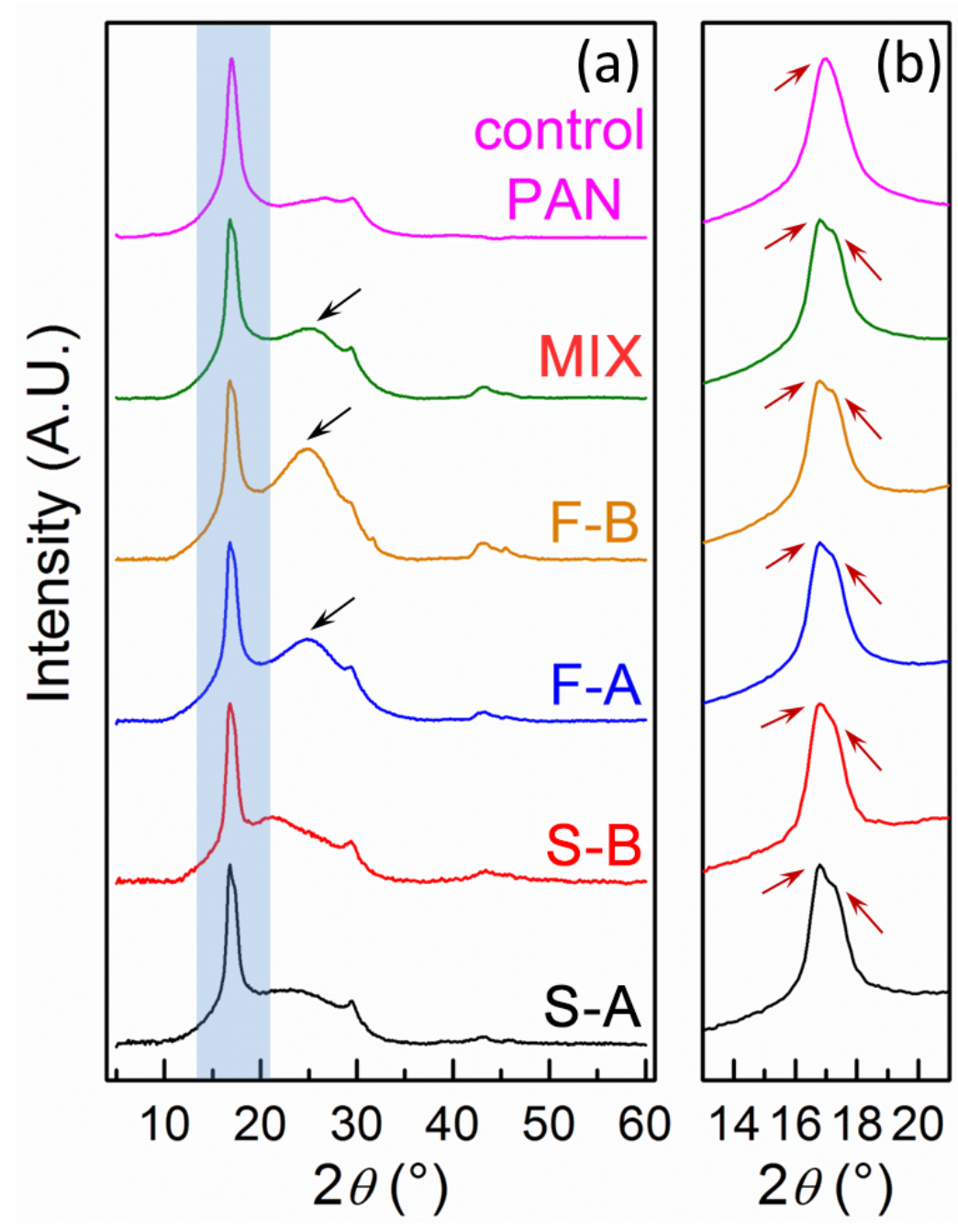

Figure 3. WAXD spectra for the as-produced (precursor) control PAN and composite films. (a) Spectra for $2 \theta=5-60^{\circ}$. (b) Spectra magnified for $2 \theta=13-21^{\circ}$. All composites exhibit a (110)/(200) doublet for the PAN major peak. The control film has a less resolved PAN peak at $\sim 17^{\circ}$. Arrows in (a) indicate the broad carbonaceous peaks. Arrows in (b) indicate the major PAN peak, where a doublet is observed in the composite samples. 
Table 2. Wide-angle X-ray (hkl) indexing, $d$-spacing, and crystal size information for PAN in each composite as well as the control film. Crystal indexing assumes lattice parameters of $a_{o}=$ $1.02 \mathrm{~nm}, b_{o}=0.61 \mathrm{~nm}$, and $c_{o}=0.51 \mathrm{~nm}[38]$.

\begin{tabular}{|c|c|c|c|c|}
\hline & & \multicolumn{3}{|c|}{ WAXD Crystal Plane } \\
\hline & & (110) & $(200)$ & (020) \\
\hline \multirow{3}{*}{ PAN/S-A } & $2 \theta\left(^{\circ}\right)$ & 16.7 & 17.3 & 29.6 \\
\hline & $d$-spacing $(\mathrm{nm})$ & 0.530 & 0.513 & 0.302 \\
\hline & crystal size (nm) & 13.2 & 9.6 & 6.1 \\
\hline \multirow{3}{*}{ PAN/S-B } & $2 \theta\left(^{\circ}\right)$ & 16.7 & 17.3 & 29.5 \\
\hline & $d$-spacing $(\mathrm{nm})$ & 0.530 & 0.514 & 0.303 \\
\hline & crystal size (nm) & 11.3 & 9.6 & 5.6 \\
\hline \multirow{3}{*}{ PAN/F-A } & $2 \theta\left(^{\circ}\right)$ & 16.7 & 17.3 & 29.5 \\
\hline & $d$-spacing $(\mathrm{nm})$ & 0.531 & 0.513 & 0.303 \\
\hline & crystal size (nm) & 10.6 & 9.0 & 6.1 \\
\hline \multirow{3}{*}{ PAN/F-B } & $2 \theta\left({ }^{\circ}\right)$ & 16.7 & 17.3 & 29.4 \\
\hline & $d$-spacing $(\mathrm{nm})$ & 0.531 & 0.513 & 0.303 \\
\hline & crystal size (nm) & 11.9 & 9.8 & 5.1 \\
\hline \multirow{3}{*}{ PAN/MIX } & $2 \theta\left({ }^{\circ}\right)$ & 16.7 & 17.3 & 29.5 \\
\hline & $d$-spacing $(\mathrm{nm})$ & 0.531 & 0.513 & 0.303 \\
\hline & crystal size $(\mathrm{nm})$ & 10.1 & 9.0 & 5.4 \\
\hline \multirow{3}{*}{ control PAN } & $2 \theta\left({ }^{\circ}\right)$ & \multirow{3}{*}{\multicolumn{2}{|c|}{$\begin{array}{c}17.0 \\
0.523 \\
6.2\end{array}$}} & 29.7 \\
\hline & $d$-spacing $(\mathrm{nm})$ & & & 0.301 \\
\hline & crystal size $(\mathrm{nm})$ & & & 3.0 \\
\hline
\end{tabular}

Figure 4 shows the fracture surfaces for the precursor films as observed by SEM. By comparing the SWNT (Figures $4 a_{1}$ and $4 b_{1}$ ) and FWNT (Figures $4 c_{1}$ and $4 d_{1}$ ) films, the thickness generally is increased by more than five times. These increases are even more pronounced for the PAN/MIX sample (Figure $4 \mathrm{e}_{1}$ ) and PAN control (Figure $4 \mathrm{f}_{1}$ ) films. This thickness variation is attributed to the dissimilarity in the resistance to the thermal shrinkage due to entropy change, which occurs during film drying. Typically polymer chains tend to coil up as the thermal energy is increased. However, it has been shown that CNT fillers can interact with and confine the polymer molecules at the interphase regions, which provides a resistance to the entropic shrinkage [43]. The differences in film shrinking behaviors suggest that PAN-CNT interfacial interactions are dependent on the CNT type. In all composites the PAN experiences some confinement as compared to the native same polymer matrix. The film thickness and thermal 
shrinkage become larger from PAN/SWNT to PAN/FWNT to PAN/MIX and to control PAN (without CNTs), indicates decreasing of filler-matrix interfacial interactions and/or confinement of the polymer chains with increased CNT diameter as well as inhomogeneity of CNTs within a sample. For example, the MIX samples consist of a mixture of SWNT, DWNT, and FWNT. It should be noted that these observed changes in interfacial interaction is directly related to the behavior of the interphase regions during pyrolysis.

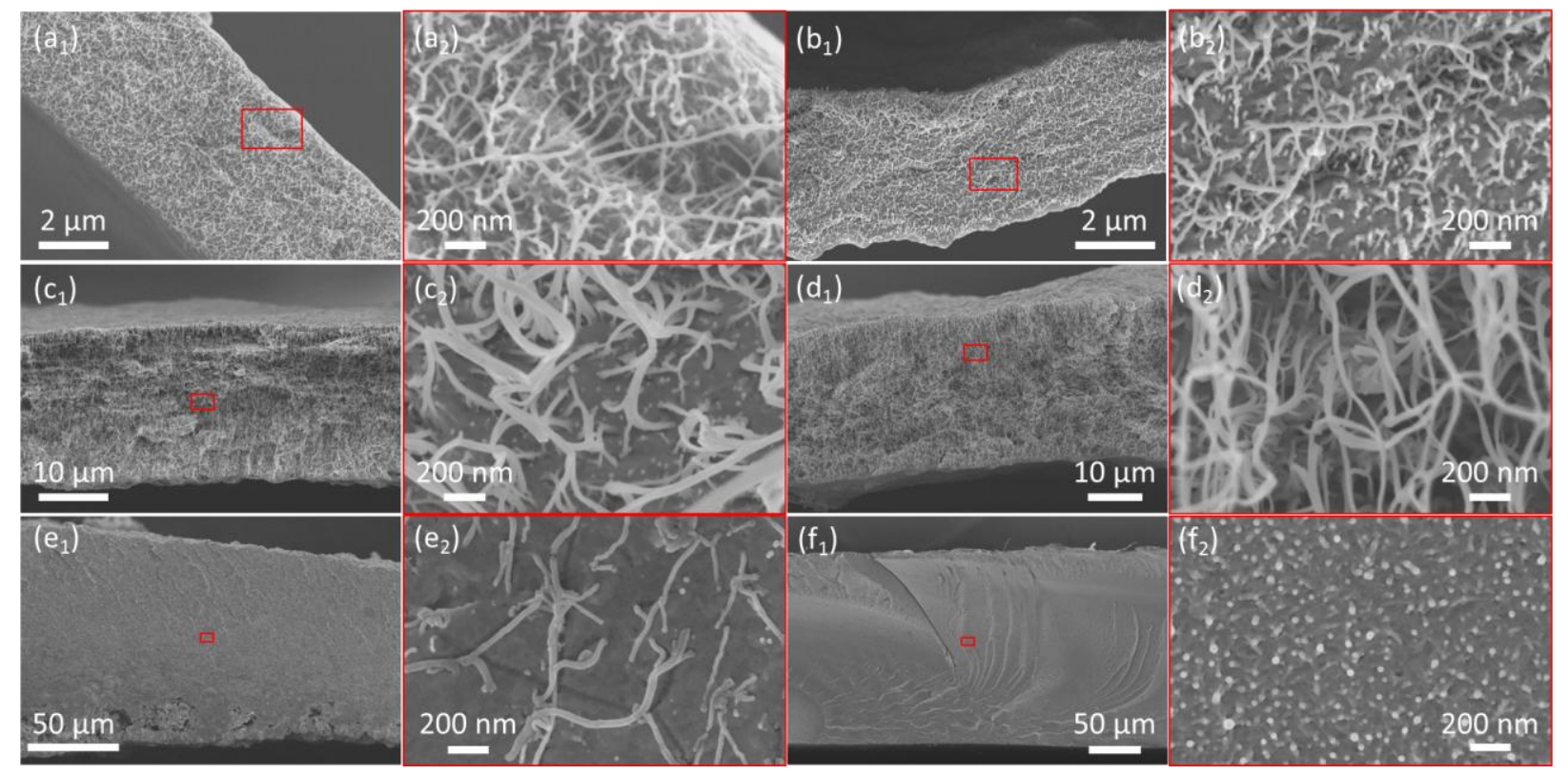

Figure 4. SEM images of the cross-section view for the as-produced (precursor) films in the order of $\left(a_{1}\right.$ and $\left.a_{2}\right)$ PAN/S-A, $\left(b_{1}\right.$ and $\left.b_{2}\right)$ PAN/S-B, $\left(c_{1}\right.$ and $\left.c_{2}\right)$ PAN/F-A, $\left(d_{1}\right.$ and $\left.d_{2}\right)$ PAN/F-B, $\left(e_{1}\right.$ and $\left.e_{2}\right)$ PAN/MIX, and $\left(f_{1}\right.$ and $\left.f_{2}\right)$ PAN control films. Images shown in $a_{2}, b_{2}, c_{2}, d_{2}, e_{2}$ and $f_{2}$ are the magnifications for the boxed regions in $\mathrm{a}_{1}, \mathrm{~b}_{1}, \mathrm{c}_{1}, \mathrm{~d}_{1}, \mathrm{e}_{1}$ and $\mathrm{f}_{1}$, respectively.

Based on image analysis of the magnified view for local regions in the composites (Figures $4 \mathrm{a}_{2}$ to $4 \mathrm{e}_{2}$ ), both the bundle size and distribution of CNTs within the samples are more uniform where SWNT is used as compared to the FWNT and MIX. In general the PAN/MIX films show 
the most non-uniform morphology in comparison to all composites. It should be noted that for all composites the interphase coating (shown in Figure 2) is not observed uniformly throughout the entire film. Therefore, the formation and thickness of $i$-PAN coating on CNTs or CNT bundles may be related to several factors including the bundle/diameter size and crystalline packing, as well as tube diameter/curvature and chirality [26, 44-45].

\subsection{Stabilized and Carbonized Structure of PAN and PAN/CNT Films}

\subsubsection{Structural Characterization using WAXD Spectroscopy}

WAXD curves for the stabilized and carbonized films are shown in Figure 5. These curves suggest that each type of CNTs interacts differently with the PAN polymer during the same stabilization and carbonization processes. This is consistent with the variations observed for interfacial interactions based on the dried film thicknesses (Figure 4) regardless of initial $i$-PAN crystallization (Figure 3). A broad peak is observed between $2 \theta$ from $23^{\circ}$ to $25^{\circ}$ (Figure 5a) due to the presence of the PAN conversion to a cyclized ladder and cross-linked structure during stabilization. The broad peak observed at $2 \theta$ of $\sim 17^{\circ}$ (see arrows in Figure 5a) indicate remnant PAN polymer in some samples due to incomplete stabilization. This residual and unreacted PAN will lead to weight loss and/or the formation of amorphous carbon phases during the subsequent carbonization steps. This peak (i.e., $\sim 17^{\circ}$ ) is most pronounced for the control PAN film, followed by the composites with MIX and FWNT loading. However, no residual PAN peak is observed for both PAN/SWNT films. 

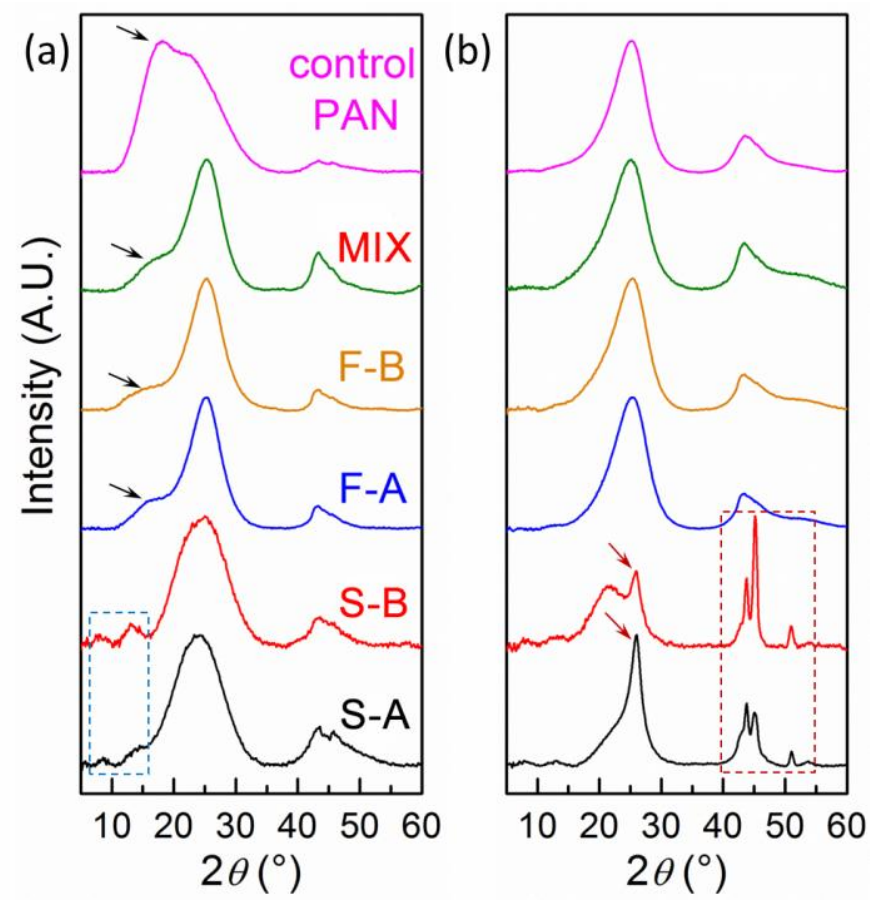

Figure 5. WAXD spectra for the (a) stabilized and (b) carbonized PAN and composite films. Stabilized films in (a) show the presence of the PAN peak at $\sim 17^{\circ}$ (see arrows), which indicate an incomplete stabilization. Carbonized films in (b) show a peak at $\sim 26^{\circ}$ indicating graphitic formation (see arrows). Additional peaks at higher $2 \theta$ positions confirm the formation of a highly ordered carbon structure for the SWNT films.

Several factors pertaining to the distribution of heat throughout the samples during treatment may contribute to a complete stabilization. A schematic representation for these parameters is provided in Figure 6. (1) Interaction within the polymer matrix as well as between the matrix and CNT fillers will vary. Considering that several CNT types are used, polymer-CNT interactions also vary in each composite. This variation in molecular interactions can largely influence the heat conduction as well as oxygen diffusion during stabilization (Figure 6a). (2) Effective heat conduction and/or dissipation in the PAN matrix highly depends on the intrinsic thermal 
properties of the CNT material, as well as variations in the dispersion quality (Figure 6b). As mentioned, dispersions in terms of bundle size, length, and distribution/aggregation, are quite different for each CNT material. (3) Film thickness of the precursors may also contribute to the under stabilization. Considering that oxidation occurring during stabilization is a diffusioncontrolled process [46-47], the inner regions of thicker films may not obtain sufficient air for a complete stabilization (Figure 6c). Similarly, the volatiles as by-products from the chemical reactions during heat treatment may not readily diffuse out. A residual PAN peak is observed for the thicker precursor films.

The pronounced PAN peak observed for the control film as compared to that in the composites may also be due to its visibly denser structure (Figure 4f) as compared to the more porous features affected by the loading of CNT fillers in the latter (Figures 4a to 4e). The variations in the stabilization process for different films may also contribute to the subsequent carbon formation during carbonization. This results in changes in the structural evolution during heating. The sufficiently stabilized structure combined with more stable interphase crystallization and confinement as a result of CNT type may be required to achieve low-temperature graphitization. 


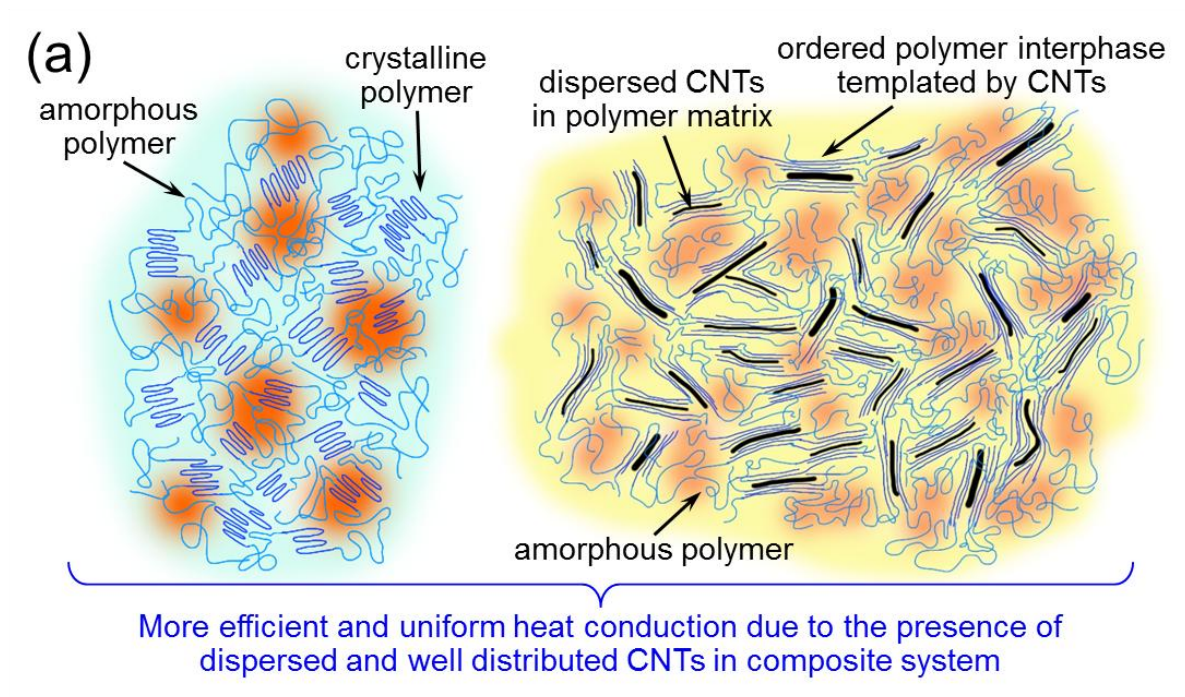

(b)

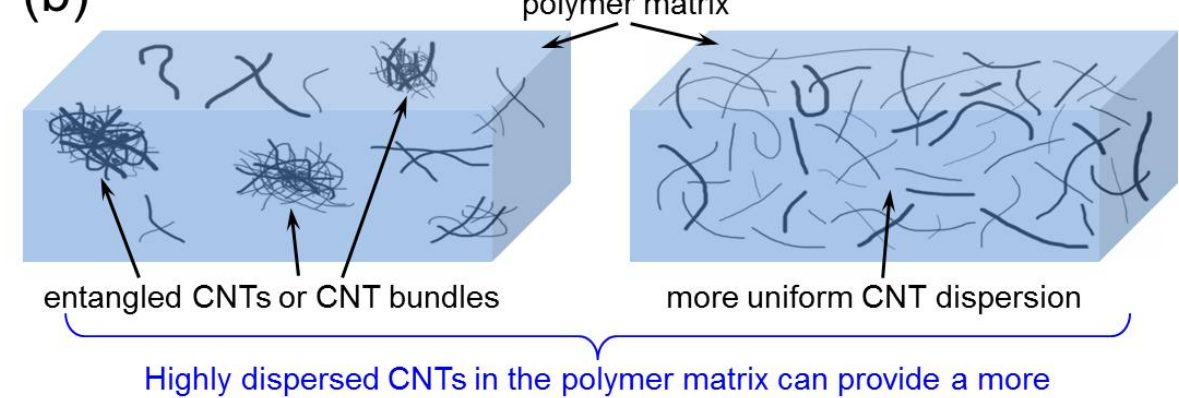

Highly dispersed CNTs in the polymer matrix can provide a more uniform path for heat conduction as well as air diffusion

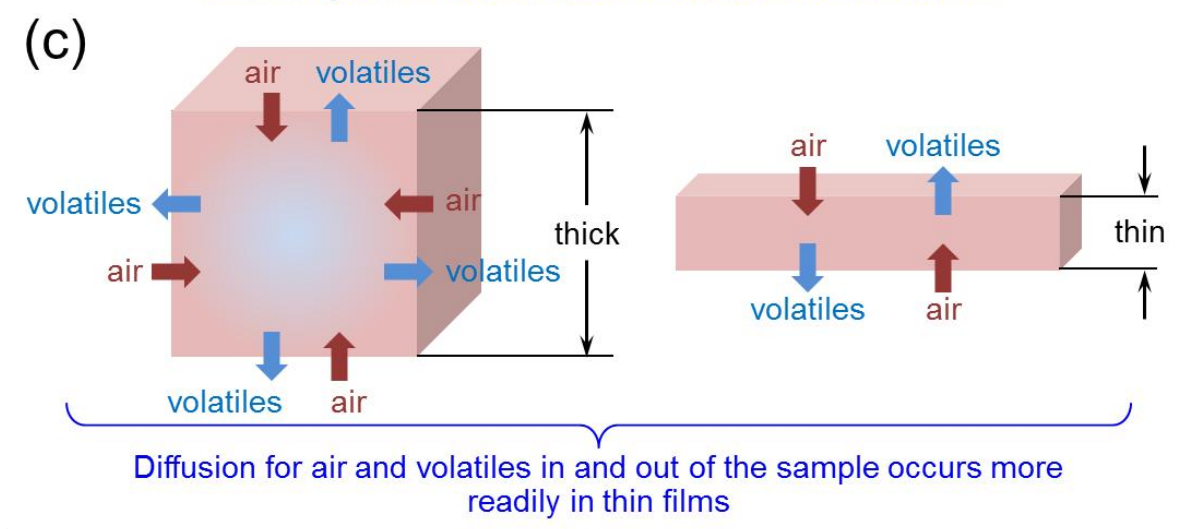

Figure 6. Schematic illustrating the potential mechanisms that lead to variations in stabilization completion for the polymer and polymer/CNT films.

The WAXD spectra for the stabilized S-A and S-B films also exhibit the signature peaks for the (10) at $\sim 8^{\circ}$ and (11) at $\sim 13^{\circ}$ reflections corresponding to the $2 \mathrm{D}$ hexagonal crystalline packing of 
SWNT (indicated by box region in Figure 5a). Due to the increased presence of carbon in the carbonized films these peaks become less pronounced. By assuming an inter-tube spacing as $0.32 \mathrm{~nm}$ [48] for SWNT bundles, the average SWNT diameter $d_{t}$ can be calculated using $d_{10}$ and $d_{11}$ for both S-A and S-B, respectively. The average calculated tube diameter $\left(d_{t}\right)$ is smaller for S-A SWNT $(0.964 \pm 0.004 \mathrm{~nm})$ than for the S-B samples $(0.969 \pm 0.079 \mathrm{~nm})$. A smaller tube diameter as well as narrower size distribution may lead to a denser packing of the pseudohexagonal 2D crystalline bundles, which can influence the formation of the interphase PAN [26, 30]. This may also contribute to the higher population and larger crystal size of the graphitic structure formed in the subsequent carbonized S-A film as compared to the S-B sample.

Overall carbonization of these various films leads to the formation of different carbon phases (Figure 5b). Only S-A and S-B composite films show predominant sharp peaks at $2 \theta$ of $\sim 26^{\circ}$ (Figure 5b) corresponding to the $d$-spacing close to the graphitic interlayer spacing (i.e., (002) peak). Additional peaks between $42^{\circ}$ and $55^{\circ}$ (indicated by dotted boxes) in Figure $5 \mathrm{~b}$ are associated with higher order peaks for crystalline graphite. The graphite (002) peaks are shown in PAN/S-A and PAN/S-B films at $26^{\circ}$ and $25.9^{\circ}$, corresponding to an average $d$-spacing of $0.342 \mathrm{~nm}$ and $0.343 \mathrm{~nm}$, respectively. The sharp peaks observed at higher $2 \theta$ angles (i.e., between $2 \theta$ from $42^{\circ}$ to $55^{\circ}$ ) (Figure 5b) are associated with two layered sequences for crystalline graphite, which are discussed in more details in Section 3.3.4.

\subsubsection{Morphological Analysis of the Carbonized Materials by SEM}

Images for the carbonized film cross-sections are shown in Figure 7. SWNT (S-A and S-B) composite films show a relatively uniform structure and exhibit a layered morphology (Figures 7a and $7 \mathrm{~b}$ ). The other CNT composites (Figures 7c, 7d and 7e) show drastic decreases in 
thickness due to large loss of PAN during carbonization (note: residual PAN remained present in stabilized sample). The carbonized control-PAN film exhibits more homogeneous and compact carbon morphology at the fractured surface (Figure 7f). In addition to material loss, with the exception of SWNT films, beaded structures are also observed (Figures $8 \mathrm{c}_{2}, 8 \mathrm{~d}_{2}$, and $8 \mathrm{e}_{2}$ ).

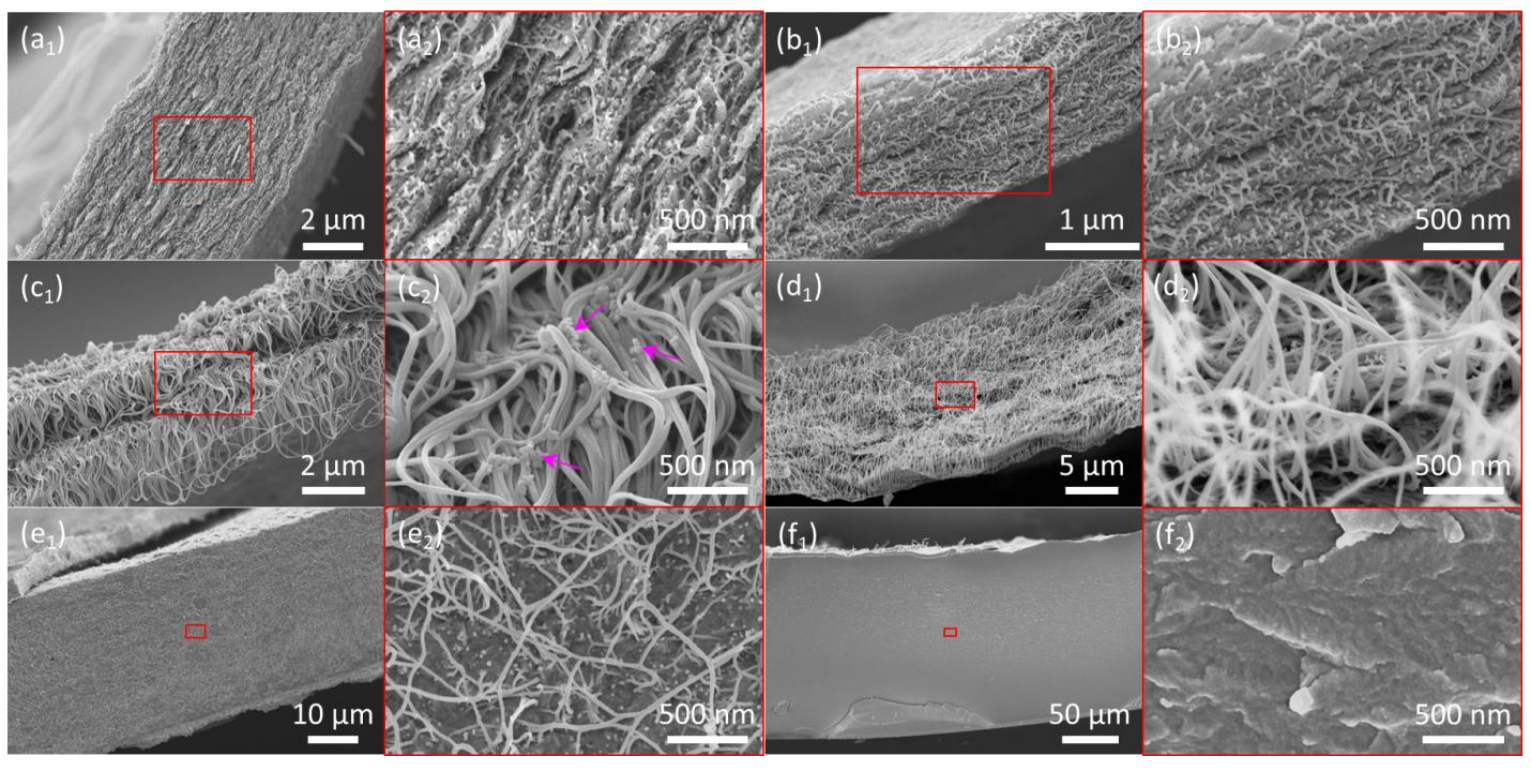

Figure 7. SEM images of the cross-section view for the carbonized films in the order of $\left(a_{1}\right.$ and

$\left.a_{2}\right)$ PAN/S-A, $\left(b_{1}\right.$ and $\left.b_{2}\right)$ PAN/S-B, $\left(c_{1}\right.$ and $\left.c_{2}\right)$ PAN/F-A, $\left(d_{1}\right.$ and $\left.d_{2}\right)$ PAN/F-B, $\left(e_{1}\right.$ and $\left.e_{2}\right)$

PAN/MIX, and $\left(f_{1}\right.$ and $\left.f_{2}\right)$ PAN control films. Images in $\mathrm{a}_{2}, \mathrm{~b}_{2}, \mathrm{c}_{2}, \mathrm{~d}_{2}, \mathrm{e}_{2}$ and $\mathrm{f}_{2}$ are magnifications for the regions within boxes marked in $\mathrm{a}_{1}, \mathrm{~b}_{1}, \mathrm{c}_{1}, \mathrm{~d}_{1}, \mathrm{e}_{1}$ and $\mathrm{f}_{1}$, respectively. Arrows in $\left(\mathrm{c}_{2}\right)$ indicate the beading morphologies present in the carbonized sample.

Figures $8 \mathrm{a}$ and $8 \mathrm{~b}$ show that for carbonized PAN/SWNT films bundles are covered or surrounded by a uniform carbon matrix, which is converted from PAN polymer. These composites also possess a more compact structure as compared to the more porous morphology observed in the other carbonized CNT composites (Figures $8 \mathrm{c}_{1}, 8 \mathrm{~d}_{1}$, and $8 \mathrm{e}_{1}$ ). As mentioned, for the F-A, F-B and MIX composites, some beaded structures are observed. These morphologies 
within the carbonized structure are associated with amorphous carbon globules (Figures $8 \mathrm{c}_{2}, 8 \mathrm{~d}_{2}$, and $8 \mathrm{e}_{2}$ ). Amorphous beading observed on the CNT surface may be indicative of potentially weaker interfacial interaction as well as insufficient confinement of the $i$-PAN by the CNTs during carbonization.

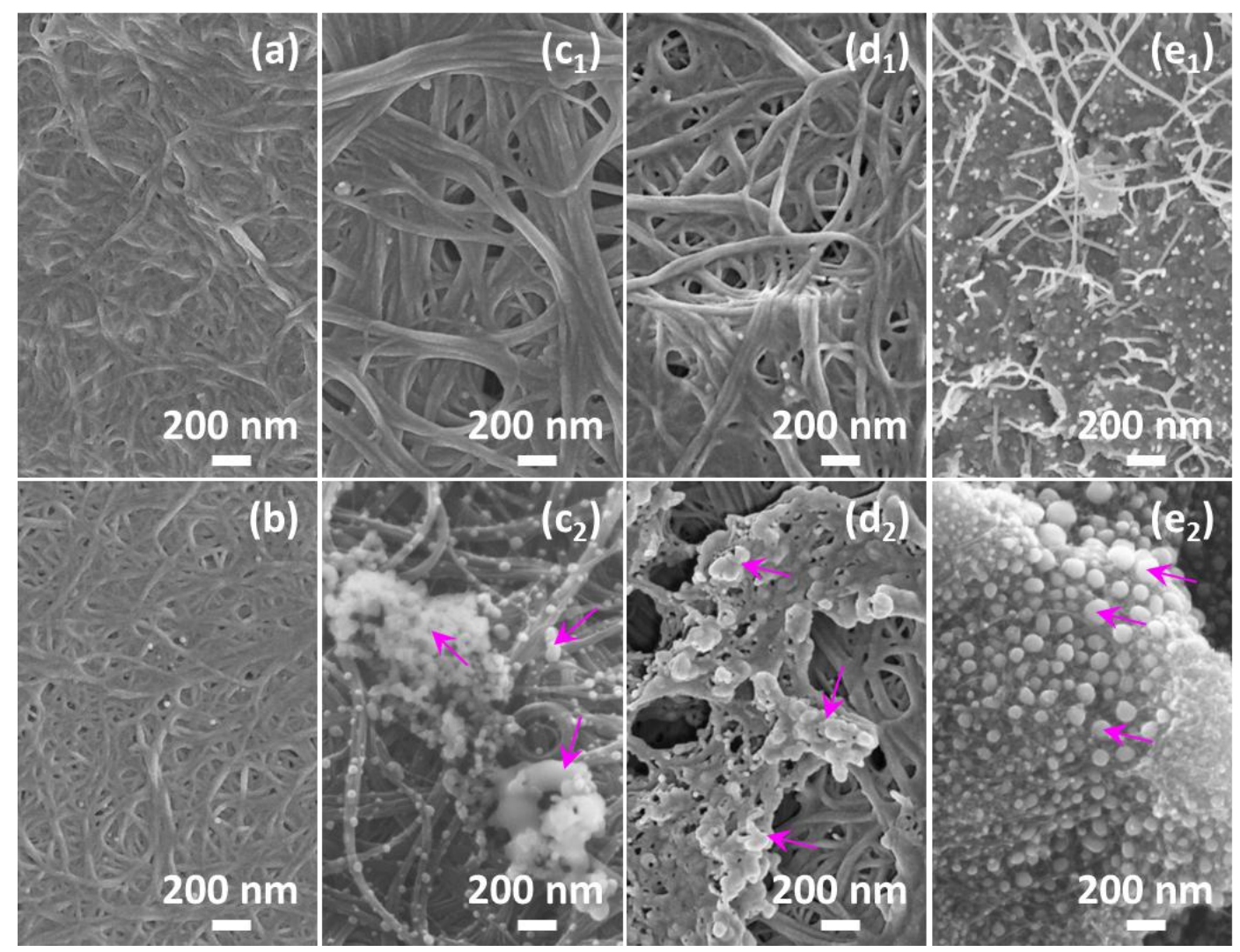

Figure 8. SEM images for the carbonized composite films in the order of (a) PAN/S-A, (b)

PAN/S-B, $\left(c_{1}\right.$ and $\left.c_{2}\right)$ PAN/F-A, $\left(\mathrm{d}_{1}\right.$ and $\left.\mathrm{d}_{2}\right)$ PAN/F-B, and $\left(\mathrm{e}_{1}\right.$ and $\left.\mathrm{e}_{2}\right)$ PAN/MIX. Low magnified images show the general film morphology (a, b, $c_{1}, d_{1}$, and $\left.e_{1}\right)$, and $\left(c_{2}, d_{2}\right.$, and $\left.e_{2}\right)$ show the regions where beaded structures are observed (see arrows).

This work demonstrates that confinement of the $i$-PAN during carbonization directly affects the formation of graphitic carbon. Previous work in carbon/CNT composites have shown that the 
glassy carbon matrix can form graphite at heat-treatment temperatures $>2500{ }^{\circ} \mathrm{C}$ due to the confinement of the matrix in the vicinity of CNT, and this is referred to as stress graphitization (i.e., induced by stresses in the surrounding matrix due to the filler presence) [49]. Similarly, with increased CNT concentration, PAN polymer graphitization was improved when heat-treated to $\geq 2000{ }^{\circ} \mathrm{C}$ due to increased confinement [50-51]. It is important to note, that these studies also show the capability of control-PAN samples (i.e., no CNT) to form graphitic structures at these high heat-treatment temperatures [50-51]. These previous works demonstrate the ability of CNT to impart confinement on the matrix and promotes stress graphitization under conditions where high temperatures can inherently promote graphite formation, comparable to commercial processes [5].

Other studies have proposed that the presence of CNTs increase the sample thermal conductivity, which may also act as catalysts or nucleation sites of graphitic crystal formation [51]. As discussed, at low carbonization temperatures (e.g., $1100{ }^{\circ} \mathrm{C}$ ) graphitic structures directly converted from PAN precursor polymer is observed at the interfacial regions [12]. However, the quantities of formed graphite are very small, and CNT loading is also low [12]. Similarly, analysis of nano-fibers fabricated by pyrolyzation of PAN/CNT electrospun fibers show only small changes in WAXD and Raman curves also indicating minimal graphitic formation in the presence of low CNT loadings [52]. In both studies, the graphitic structures were only observed by HR-TEM at localized regions $[12,52]$. The findings in this current work differs from the previous studies, in that (1) the interphase polymer formation is intentional, and (2) the precursor PAN graphitizes (i.e., (002) peak in XRD spectra) at $1100{ }^{\circ} \mathrm{C}$, which does not facilitate graphitic formation for PAN in commercial processes. 
Based on the observations in this work as well as the previous studies, the formation of graphite from this interphase precursor PAN is influenced by the confinement of the chains in the vicinity of the nanotubes. However, each nanotube type is shown to have a different confinement effect for the PAN polymer. In general, as the nanotube diameter increases, the confinement effect is observed to decrease when considering thermal shrinkage of the matrix during drying and pyrolysis (Figures 4 and 8). Previous studies have shown that the PAN extended-chain conformation can be induced due to CNT confinement [53]. It is believed that the CNT diameter closer to the polymer extended-chain diameter (i.e., ranging from 0.6 to $0.8 \mathrm{~nm}$, due to conformational variations between planer zig-zag and helical arrangements) plays a larger role in templating alignment and preventing shrinkage during thermal treatment. It is very important for the PAN to maintain its extended-chain conformation to increase cyclized structure formation during stabilization. This structural presence can promote the formation of the graphitic sheets during carbonization. Typically, commercial PAN precursor fibers are semi-crystalline and consisting mainly of both folded-chain crystals and amorphous regions. For this reason, the cyclized structure formed during stabilization is not continuous and the resultant carbonized structure is mostly turbostratic (i.e., short, wavy, and defective carbon sheets), resulting in a broad carbon peak in the WAXD spectra [1].

This work shows that tailored control of the interphase region and the polymer chain conformation [30] can influence early formation of large graphitic domains at low pyrolysis temperatures. From some previous studies, large diameter CNT types (e.g., MWNT) were used to induce graphitic formation. However there are two major distinctions for these works as compared to the current study. (i) The confined matrix (i.e., glassy carbon) consists of domain sizes that are considerable larger than the individual PAN chains [49-50], and (ii) graphitization 
temperatures comparable to the commercial approaches are used in the heat-treatment processes [49-51]. These large diameter tubes are not effective in this current study. Similar to the previous work, it is important to note that size considerations in terms of the matrix material and CNT type can affect confinement and templating, which determine the temperature and conditions for stress-induced graphitization in the interfacial regions. This study has shown that SWNT is more effective in confining the polymer chain to promote stress-induced graphitization, due to the similar size-scale of SWNTs and PAN molecules.

\subsubsection{Graphitic Structure Observed by High Resolution TEM}

High resolution TEM (HR-TEM) imaging and lattice spacing measurements of the carbonized films were performed in addition to WAXD spectra to characterize carbon formation. Figures 9 and 10 provide images at both low and high magnifications for each carbonized composite film. Graphitic structures are observed in all cases. Based on analysis of several images taken for each sample, the carbonized PAN/S-A and PAN/S-B show the most prevalent formation of graphite ribbons, and this is not found for the other carbonized films. The graphitic ribbons formed in S-A composite (Figure 9a) are visibly much longer and thicker, as compared to the S-B composite (Figure 9b). Based on HR-TEM analysis, it was found that graphitic ribbons in the S-A samples show up to 35 layers, while S-B films show up to 15 layers. From the WAXD analysis, crystal thickness $\left(L_{L}\right)$ for the graphitic regions was on average $4.5 \mathrm{~nm}$ for both samples (i.e., corresponding to 13 to 15 layers). Both the stack/layer thickness and length of the carbon ribbons present in the carbonized S-A and S-B samples demonstrate the formation of graphite at low temperature. Turbostratic carbon, typically formed in this temperature range, normally exhibits a stack thickness of $\sim 1 \mathrm{~nm}$ (i.e., 3 to 4 layers) [54]. 
Based on this HR-TEM analysis, the graphitic structures in the S-A and S-B SWNT samples also exhibit much larger crystal size as compared to the composites with the other CNT materials (Figures 10a to 10c). These results are also in accordance with the XRD analysis. WAXD spectra for carbonized F-A, F-B, and MIX composites do not show a (002) peak. However, TEM analysis indicates the presence of graphite. These results are not contradictory; instead they show that the prevalence of graphite is small for the F-A, F-B, and MIX composites. In comparison, amorphous and turbostratic carbonaceous structures dominate the materials.

HR-TEM imaging enables measurements of the lattice spacing for the observed graphitic ribbons. These spacings are labeled for each composite (Figures 9 and 10). The averaged lattice spacing observed by HR-TEM is $0.342 \pm 0.005 \mathrm{~nm}$ for S-A and $0.343 \pm 0.005 \mathrm{~nm}$ for S-B (Figure 9), which is consistent with the XRD results. In addition, the measured spacings for F-A, F-B and MIX samples (labeled in Figure 10) increase as compared to the SWNT composites, which indicates the presence of a more defective/disordered graphitic structure (i.e., turbostratic graphite). 


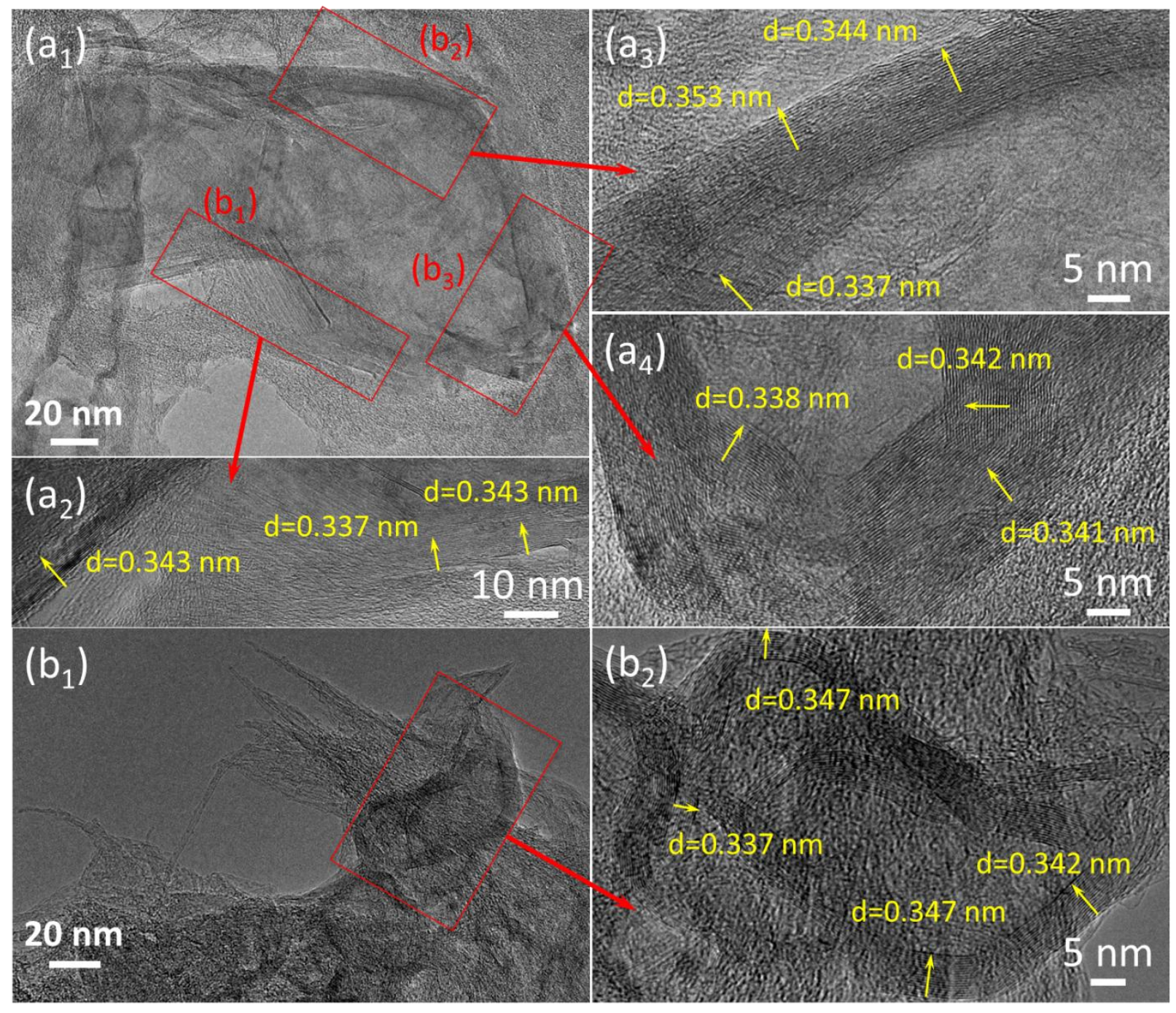

Figure 9. HR-TEM images at low and high magnifications are provided for $\left(\mathrm{a}_{1}, \mathrm{a}_{2}, \mathrm{a}_{3}\right.$, and $\left.\mathrm{a}_{4}\right)$ PAN/S-A and $\left(b_{1}\right.$ and $\left.b_{2}\right)$ PAN/S-B. 


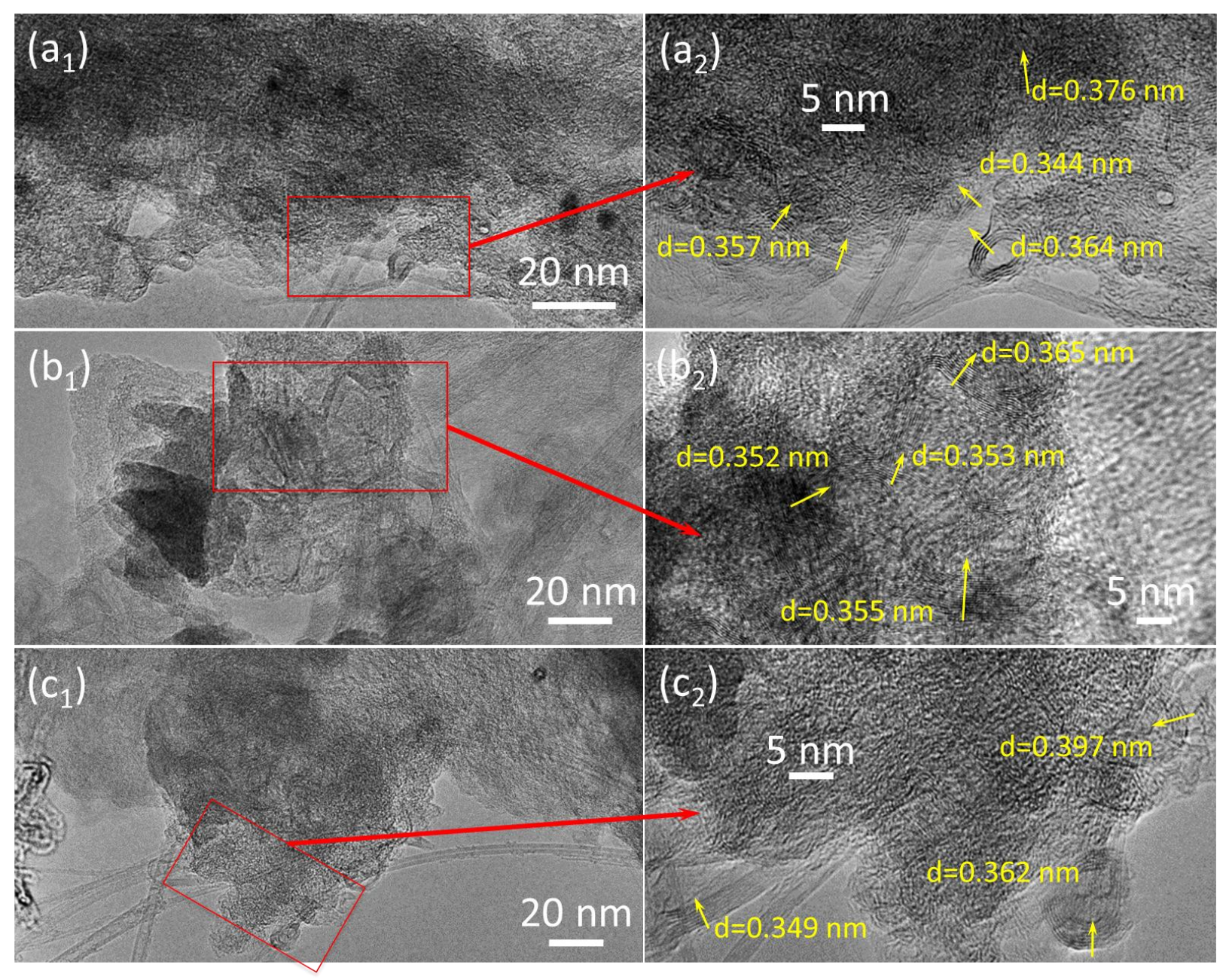

Figure 10. HR-TEM images at low and high magnifications are provided for $\left(a_{1}\right.$ and $\left.a_{2}\right)$ PAN/F$\mathrm{A},\left(\mathrm{b}_{1}\right.$ and $\left.\mathrm{b}_{2}\right)$ PAN/F-B and $\left(\mathrm{c}_{1}\right.$ and $\left.\mathrm{c}_{2}\right)$ PAN/MIX samples.

\subsubsection{Crystallographic Analysis of the Graphite Formed in SWNT Composites}

A detailed analysis is performed to understand the structural forms of the graphite present in the SWNT samples. The observation of the sharp peaks at higher $2 \theta$ positions $\left(42^{\circ}\right.$ to $\left.55^{\circ}\right)$ for both S-A and S-B composites (Figure 11a) suggests highly crystalline perfection of the layered graphitic structure. The WAXD peaks ranging from $42^{\circ}$ to $55^{\circ}$ for both SWNT loaded samples correspond to two layered graphitic crystal structures (Figures $11 b_{1}$ and $11 b_{2}$ ). The crystal planes 
are indexed accordingly in Figure 11a. Table 3 lists the WAXD crystal peak information including peak position and the corresponding indexed plane with respect to each structure. Table 4 provides the $d$-spacing and crystal size for each peak.

The variation in stacking order for the graphene layers distinguishes both graphite crystal structures [55]. Ordered graphite typically exhibits a hexagonal structure (Figure $\left.6 b_{1}\right)$ where graphene layers have ABAB stacking (known as Bernal stacking) along the c-axis. This is called the hexagonal 2H-graphite with the space group P63/mmc (i.e., No. 194) [56-57]. In addition, graphite in a rhomohedral structure (Figure $11 \mathrm{~b}_{2}$ ) with ACBACB stacking is also possible. This structure is called the rhomohedral 3R-graphite with the space group R3 (i.e., No. 146) [56-57]. The hexagonal unit cell contains six atoms, while the rhombohedral unit cell only contains two atoms. This rhombohedral unit cell can also be considered as a larger hexagonal unit cell with the lattice constants $\left(a_{o}=0.246 \mathrm{~nm}\right.$, but $\left.c_{o}=1.004 \mathrm{~nm}\right)$. The $c_{o}$-axis is larger as compared to the ABAB hexagonal crystal (i.e., $c_{o}=0.671 \mathrm{~nm}$ ) (Figure $\left.11 \mathrm{~b}_{2}\right)$. 

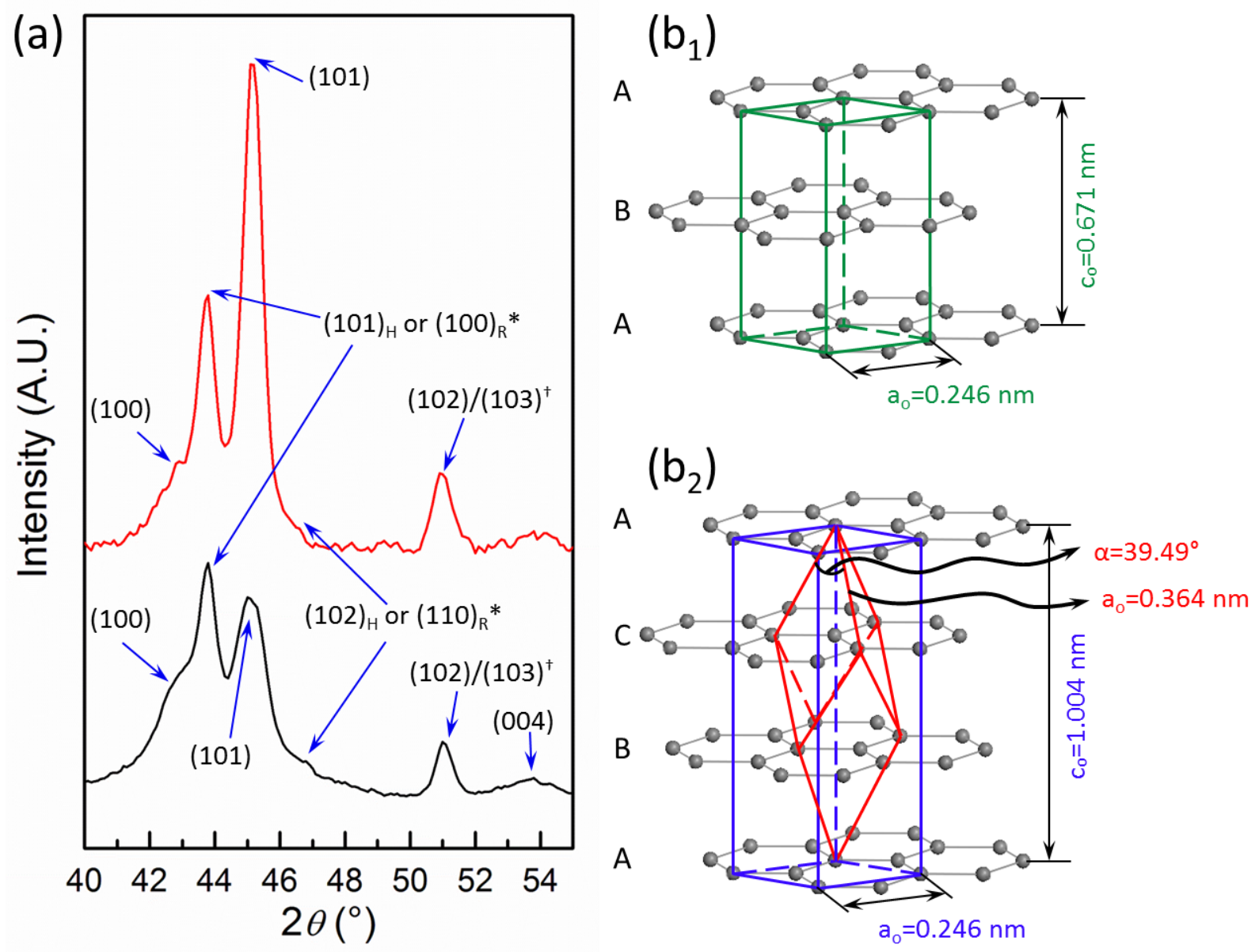

Figure 11. (a) WAXD spectra for the carbonized S-A and S-B composite films at higher $2 \theta$ regions between $40^{\circ}$ to $55^{\circ}$. Based on the observed crystal peaks two graphite crystal structures are identified and distinguished by layer stacking. Peaks in (a) corresponding to graphite crystal structures are indexed and labeled. $\left(\mathrm{b}_{1}\right)$ ABAB layered hexagonal $(2 \mathrm{H})$ graphite with lattice parameters of $a_{o}=b_{o}=0.246 \mathrm{~nm}$ and $c_{o}=0.671 \mathrm{~nm} .\left(\mathrm{b}_{2}\right)$ ACBACB layered rhombohedral (3R) graphite with lattice parameters $a_{o}=b_{o}=c_{o}=0.364 \mathrm{~nm}$ and $\alpha=\beta=\gamma=39.49^{\circ}$, which is equivalent to a hexagonal unit cell (shown in blue) with $a_{o}=b_{o}=0.246 \mathrm{~nm}$ and $c_{o}=1.004 \mathrm{~nm}[58-59] .{ }^{*} \mathrm{H}$ and R represent the larger hexagonal unit cell and the rhombohedral unit cell, respectively, shown in $\left(b_{1}\right)$ and $\left(\mathrm{b}_{2}\right) .{ }^{\dagger}$ Peaks at $\sim 51^{\circ}$ can be indexed as (102) in (ABAB) hexagonal unit cell or (103) in (ACBACB) hexagonal unit cell. 
Although the 3R-graphite structure is considered less stable than the $2 \mathrm{H}$-graphite from the energy point of view, the total energy for the former differs only slightly from the latter (i.e., the more stable Bernal $\mathrm{ABAB}$ stacking) [56]. For this reason, graphite crystals typically possess stacking faults where the ACBACB stacking is admixed with the ABAB stacking. Stacking faults present in graphite usually do not affect the in-plane lattice parameters (i.e., $a_{o}$ and $b_{o}$ ) due to the strong $\mathrm{C}-\mathrm{C}$ covalent bonds. However, they can largely affect the weak interplanar bonding, resulting in the increase in the interlayer distance [56]. Therefore, when the stacking is not correlated to any order ( $\mathrm{AB}$ or $\mathrm{ACB}$ ) and completely random, the structure becomes the aforementioned 'turbostratic graphite'.

Table 3. Theoretical and experimental peak positions and indexing for the layered graphite crystal unit cells found in S-A and S-B carbonized films based on WAXD analysis. The crystal planes are indexed by assuming a hexagonal $(2 \mathrm{H})$ graphite unit cell with lattice parameters of $a_{o}=b_{o}=0.246 \mathrm{~nm}$ and $c_{o}=0.671 \mathrm{~nm}$, and a rhomohedral (3R) graphite unit cell with lattice parameters $a_{o}=b_{o}=c_{o}=0.364 \mathrm{~nm}$ and $\alpha=\beta=\gamma=39.49^{\circ}$, which is equivalent to a larger hexagonal unit cell with $a_{o}=b_{o}=0.246 \mathrm{~nm}$ and $c_{o}=1.004 \mathrm{~nm}$.

\begin{tabular}{|c|c|c|c|c|c|}
\hline \multicolumn{2}{|c|}{ experimental $2 \theta\left(^{\circ}\right)$} & \multirow{3}{*}{$\begin{array}{c}\text { theoretical } \\
2 \theta\left(\left(^{\circ}\right)\right.\end{array}$} & \multicolumn{3}{|c|}{ indexed planes for different crystal unit cells } \\
\hline \multirow{2}{*}{$\begin{array}{c}\text { carbonized } \\
\text { PAN/S-A }\end{array}$} & \multirow{2}{*}{$\begin{array}{c}\text { carbonized } \\
\text { PAN/S-B }\end{array}$} & & \multirow{2}{*}{$\begin{array}{c}\text { (ABAB) } \\
\text { hexagonal }\end{array}$} & \multicolumn{2}{|c|}{$(\mathrm{ACBACB})$} \\
\hline & & & & hexagonal & rhombohedral \\
\hline 42.8 & 42.8 & 42.5 & $(100)$ & - & - \\
\hline 43.8 & 43.7 & 43.5 & - & $(101)$ & $(100)$ \\
\hline 45.1 & 45.1 & 44.7 & $(101)$ & - & - \\
\hline 46.6 & 46.3 & 46.3 & - & $(102)$ & $(110)$ \\
\hline 51.0 & 51.0 & 50.8 & $(102)^{\dagger}$ & $(103)^{\dagger}$ & - \\
\hline 53.7 & 54.0 & 54.7 & $(004)$ & - & - \\
\hline
\end{tabular}

${ }^{\dagger}$ Peaks at $51^{\circ}$ can be indexed as (102) in (ABAB) hexagonal unit cell or (103) in (ACBACB) hexagonal unit cell. 
Table 4. Experimental peak positions, $d$-spacing, and crystal size information for the graphite formed in the carbonized PAN/P and PAN/SP composites from WAXD information.

\begin{tabular}{c|c|c|c|c|c|c|c|c}
\hline \multirow{3}{*}{ PAN/S-A } & $2 \theta\left(^{\circ}\right)$ & 26.0 & 42.8 & 43.8 & 45.1 & 46.6 & 51.0 & 53.7 \\
& $d$-spacing $(\mathrm{nm})$ & 0.342 & 0.211 & 0.206 & 0.201 & 0.195 & 0.179 & 0.170 \\
& crystal size (nm) & 4.7 & 5.7 & 12.6 & 8.1 & 6.9 & 16.8 & 5.0 \\
\hline \multirow{3}{*}{ PAN/S-B } & $2 \theta\left({ }^{\circ}\right)$ & 25.9 & 42.8 & 43.7 & 45.1 & 46.3 & 51.0 & 54.0 \\
& $d$-spacing $(\mathrm{nm})$ & 0.343 & 0.211 & 0.207 & 0.201 & 0.196 & 0.179 & 0.170 \\
& crystal size (nm) & 4.0 & 7.9 & 14.1 & 12.5 & 6.6 & 14.0 & 5.5 \\
\hline
\end{tabular}

\subsubsection{Thermogravimetric Analysis of the Carbonized Films}

TGA analysis was performed for all the carbonized composite films as well as the neat-CNT buckypapers. The degradation behaviors of the buckypapers (Figure 12a) are related to the intrinsic characteristics of the CNTs used. Raman analysis for the G-band and D-band (i.e. $I_{G / D}$ ratio) suggests variations in the graphitic structure quality for different CNT materials used. Based on the Raman data, generally it was found that the SWNT exhibit more defective structure, followed by MIX, and subsequently both FWNT batches. The defects or disorders present in the graphitic walls tend to result in oxidative instability promoting tube unzipping and degradation through these sites [60]. In addition, since SWNT consists of only a single layer of graphene. Therefore, the degradation process may occur earlier than the FWNT samples with more layers present. The presence of metal catalysts can also potentially catalyze the oxidation process and reduce the degradation threshold. This process may contribute to the lower and broader degradation temperature range observed for S-A SWNT (containing more metallic impurities), as compared to the other CNTs. 
For the carbonized films (Figures 12b), the degradation temperature was improved for all CNTs with the exception of the MIX samples (Figures 12c and 12d). The improvement in thermal stability (i.e., the degradation temperature) for the different CNT materials is varied. Large increases in the degradation temperatures for both SWNT systems are observed, and relatively small improvements are found for both FWNT composites (Figures 12c and 12d).

The thermal degradation may also be related to the thermal conductivity of the material, which is dominated by phonon transport within the graphitic basal planes. Within the material structure, thermal conductivity or phonon transport can be increased by improving the orientation with respect to the basal plane, as well as reducing transport obstacles such as pores, grain boundaries, and in-plane lattice defects in the graphite [61]. The S-A SWNT samples show the largest improvement in the thermal stability due the presence of the most prevalent graphite structure, which is consistent with the observation by microscopy and spectroscopy, and this is followed by the S-B samples (Figure 12d). S-A, S-B, F-A, and F-B samples all show and increase in the degradation temperatures. However, for F-A and F-B this increase is small, which may be due to noticeably less graphitic formation in these materials. The MIX samples show a decrease in the degradation temperature, which is related to the formation of a large population of amorphous carbon beads in carbonized films (Figure 8). In general, the changes in the degradation behaviors can be related to the morphology variations in all the carbonized composite films. 
(a)

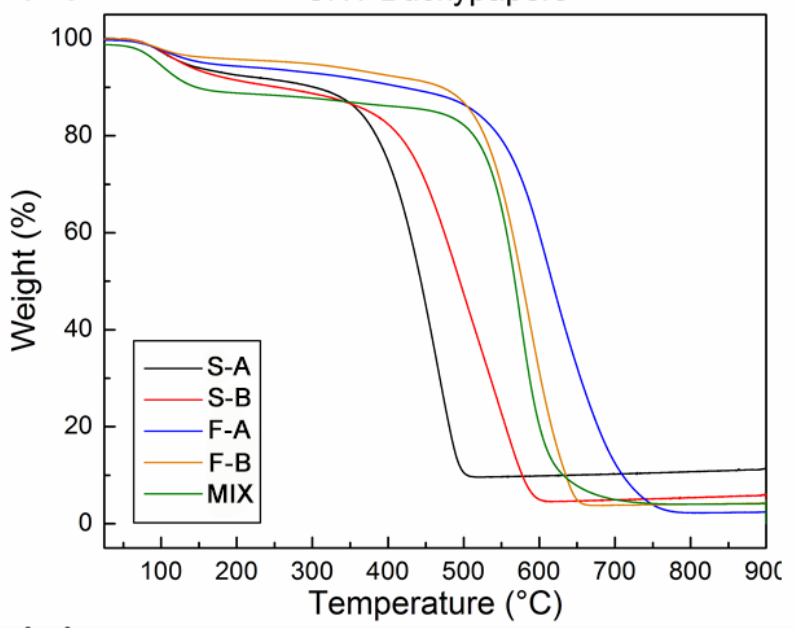

(c)

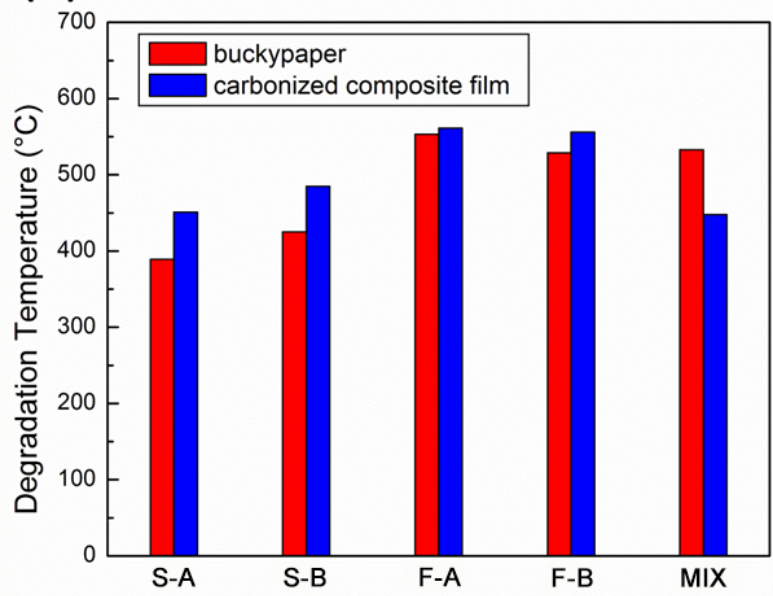

(b)

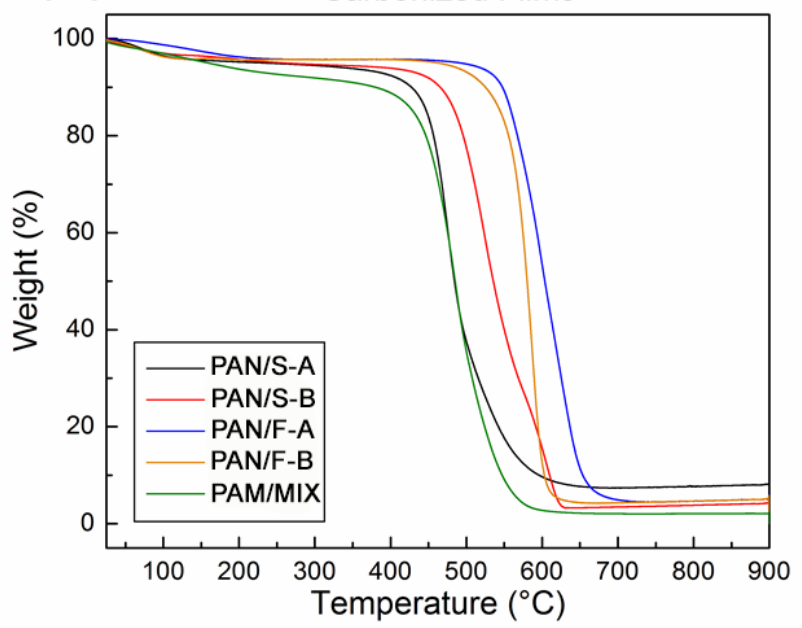

(d)

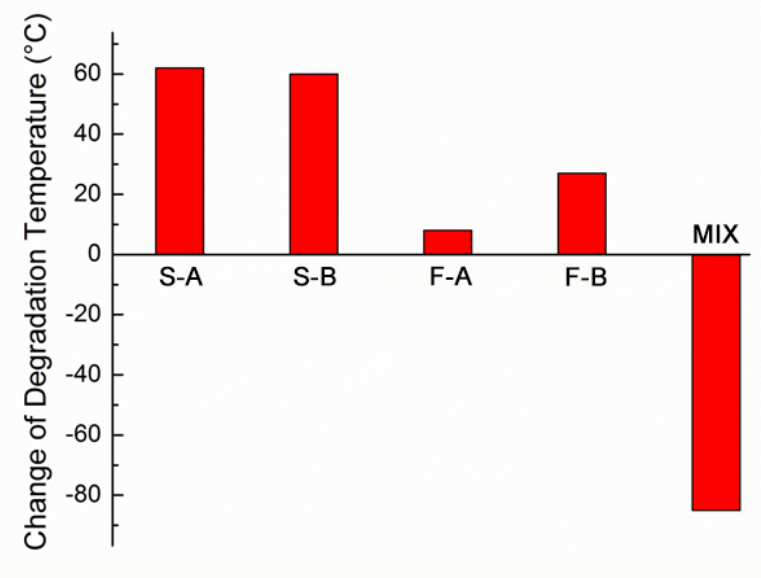

Figure 12. TGA weight percentage versus temperature curves for (a) CNT buckypapers and (b)

the carbonized composite films. (c and d) Bar charts show the degradation temperature and changes between CNT buckypapers as compared to the PAN/CNT carbonized films.

\subsubsection{Graphitization of The Carbonized PAN/S-A Film}

Carbonized PAN/S-A films showed the highest prevalence for graphitic ribbons at low temperature. This sample was further heat treated to $2100{ }^{\circ} \mathrm{C}$ for 40 minutes, to observe any additional structural evolution at graphitization temperatures. Figure 13 shows the WAXD curves for the PAN/S-A samples before and after treatment at $2100{ }^{\circ} \mathrm{C}$. Several changes were 
observed: (1) the amorphous carbon peak is significantly diminished and transformed to graphite at higher temperature, (2) higher order peaks associated with rhombohedral and hexagonal carbon are removed indicating a conversion to larger graphite sheets, and (3) the in-plane graphite peak is prevalent.

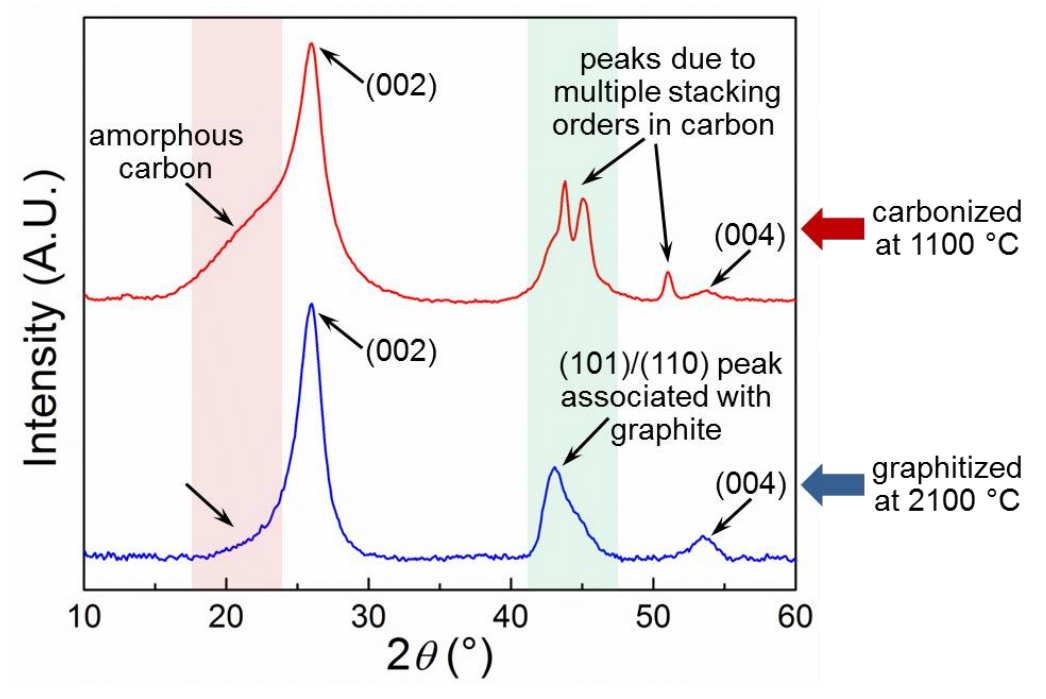

Figure 13. WAXD spectra for carbonized and graphitized PAN/S-A film. After graphitization, (i) the presence of amorphous carbon is largely reduced, (ii) graphitic (002) and (004) peaks become more pronounced, (iii) peaks related to multiple stacking order of graphene layers disappear, and (iv) the (101)/(110) peak associated with graphite is dominant.

\section{CONCLUSIONS}

This study demonstrates that confinement of the PAN molecules in an interphase structures around CNT materials may lead to early onset of graphitic formation at $1100^{\circ} \mathrm{C}$. This graphitic structure is more prevalent in samples containing SWNT, where the nanotube diameter is smallest. The low temperature graphite formed consists of two distinct layer crystals belonging to the rhomobohedral and hexagonal packing. This structure may be healed to further yield large 
domains of hexagonal graphite after heat treatment up to $2100^{\circ} \mathrm{C}$. As evidence by SEM, FWNT and MIX samples show prevalence of amorphous carbon, where beaded structures are observed on the surface of the CNTs. This implies that the interphase polymer is not confined during heattreatment by the nanotube, leading to polymer coiling/shrinkage. Such structures were not observed for the SWNT samples. This demonstrated the ability of SWNT to template a crystalline PAN interphase, which is required to form low temperature graphite. Thermal degradation behavior of the carbonized PAN/CNT samples is also consistent with the degree of graphitic formation observed. The recognition and understanding for the formation of this low temperature graphitic phase in the presence of CNTs has important implications toward processing of CFs based on PAN precursor materials.

\section{ACKNOWLEDGEMENTS}

Funding support for this work is provided by DARPA and the Army Research Office (ARO) (W911NF-13-1-0190), as well as the Air Force Office of Scientific Research (AFOSR) (FA9550-11-1-0153). The authors would also like to thank Professor Philip Bradford at North Carolina State University for his assistance in performing high-temperature graphitization treatment for the PAN/CNT samples.

\section{REFERENCES}

[1] Chung Deborah D. L. Carbon Fiber Composites. Butterworth-Heinemann: Newton, 1994. [2] Morgan Peter, Carbon Fibers and Their Composites. Taylor \& Francis Group: Boca Raton, 2005.

[3] Chand S. Review Carbon fibers for composites. Journal of Materials Science 2000;35(6):1303-1313. 
[4] Liu Yaodong, Kumar Satish. Recent Progress in Fabrication, Structure, and Properties of Carbon Fibers. Polym. Rev. 2012;52(3-4):234-258.

[5] Minus Marilyn, Kumar Satish. The processing, properties, and structure of carbon fibers. Jom 2005;57(2):52-58.

[6] Rahaman M. S. A., Ismail A. F., Mustafa A. A review of heat treatment on polyacrylonitrile fiber. Polymer Degradation and Stability 2007;92(8):1421-1432.

[7] Frank Erik, Hermanutz Frank, Buchmeiser Michael R. Carbon Fibers: Precursors, Manufacturing, and Properties. Macromol. Mater. Eng. 2012;297(6):493-501.

[8] Watt W., Johnson W. Mechanism of Oxidisation of Polyacrylonitrile Fibers. Nature 1975;257(5523):210-212.

[9] Rangarajan P., Bhanu V. A., Godshall D., Wilkes G. L., McGrath J. E., Baird D. G. Dynamic oscillatory shear properties of potentially melt processable high acrylonitrile terpolymers. Polymer 2002;43(9):2699-2709.

[10] Fitzer E., Müller D. J. The influence of oxygen on the chemical reactions during stabilization of pan as carbon fiber precursor. Carbon 1975;13(1):63-69.

[11] Chae H. G., Choi Y. H., Minus M. L., Kumar S. Carbon nanotube reinforced small diameter polyacrylonitrile based carbon fiber. Composites Science and Technology 2009;69(3-4):406-413. [12] Chae H. G., Minus M. L., Rasheed A., Kumar S. Stabilization and Carbonization of Gel Spun Polyacrylonitrile/Single Wall Carbon Nanotube Composite Fibers. Polymer 2007;48(13):3781-3789.

[13] Liu Yaodong, Chae Han Gi, Kumar Satish. Gel-spun carbon nanotubes/polyacrylonitrile composite fibers. Part II: Stabilization reaction kinetics and effect of gas environment. Carbon 2011;49(13):4477-4486.

[14] Liu Yaodong, Chae Han Gi, Kumar Satish. Gel-spun carbon nanotubes/polyacrylonitrile composite fibers. Part I: Effect of carbon nanotubes on stabilization. Carbon 2011;49(13):44664476.

[15] Bashir Z. A critical review of the stabilisation of polyacrylonitrile. Carbon 1991;29(8):10811090.

[16] Yu Meijie, Wang Chengguo, Bai Yujun, Zhu Bo, Ji Minxia, Xu Yong. Microstructural evolution in polyacrylonitrile fibers during oxidative stabilization. Journal of Polymer Science Part B: Polymer Physics 2008;46(7):759-765.

[17] Ji Minxia, Wang Chengguo, Bai Yujun, Yu Meijie, Wang Yanxiang. Structural Evolution of Polyacrylonitrile Precursor Fibers during Preoxidation and Carbonization. Polymer Bulletin 2007;59(4):527-536.

[18] Fazlitdinova A. G., Tyumentsev V. A., Podkopayev S. A., Shveikin G. P. Changes of polyacrylonitrile fiber fine structure during thermal stabilization. Journal of Materials Science 2010;45(15):3998-4005.

[19] Devasia Renjith, Nair C. P. Reghunadhan, Sadhana R., Babu N. S., Ninan K. N. Fourier transform infrared and wide-angle X-ray diffraction studies of the thermal cyclization reactions of high-molar-mass poly(acrylonitrile-co-itaconic acid). J. Appl. Polym. Sci. 2006;100(4):30553062.

[20] Yu Meijie, Wang Chengguo, Zhao Yaqi, Zhang Min, Wang Weiqiang. Thermal properties of acrylonitrile/itaconic acid polymers in oxidative and nonoxidative atmospheres. J. Appl. Polym. Sci. 2010;116(2):1207-1212. 
[21] Fitzer E., Frohs W., Heine M. Optimization of stabilization and carbonization treatment of PAN fibres and structural characterization of the resulting carbon fibres. Carbon 1986;24(4):387395.

[22] Ouyang Qin, Cheng Lu, Wang Haojing, Li Kaixi. Mechanism and kinetics of the stabilization reactions of itaconic acid-modified polyacrylonitrile. Polymer Degradation and Stability 2008;93(8):1415-1421.

[23] Chae Han Gi, Minus Marilyn L., Kumar Satish. Oriented and exfoliated single wall carbon nanotubes in polyacrylonitrile. Polymer 2006;47(10):3494-3504.

[24] Green Emily C., Zhang Yiying, Minus Marilyn L. Understanding the effects of nanocarbons on flexible polymer chain orientation and crystallization: Polyethylene/carbon nanochip hybrid fibrillar crystal growth. J. Appl. Polym. Sci. 2014;131(18):9235-9245.

[25] Meng Jiangsha, Zhang Yiying, Song Kenan, Minus Marilyn L. Forming Crystalline Polymer-Nano Interphase Structures for High-Modulus and High-Tensile/Strength Composite Fibers. Macromol. Mater. Eng. 2014;299(2):144-153.

[26] Minus M. L., Chae H. G., Kumar S. Single Wall Carbon Nanotube Templated Oriented Crystallization of Poly(vinyl alcohol). Polymer 2006;47(11):3705-3710.

[27] Minus M. L., Chae H. G., Kumar S. Interfacial Crystallization in Gel-Spun Poly(vinyl alcohol)/Single-Wall Carbon Nanotube Composite Fibers. Macromolecular Chemistry and Physics 2009;210(21):1799-1808.

[28] Minus Marilyn L., Chae Han Gi, Kumar Satish. Polyethylene crystallization nucleated by carbon nanotubes under shear. ACS Applied Materials \& Interfaces 2012;4(1):326-330.

[29] Song Kenan, Zhang Yiying, Meng Jiangsha, Minus Marilyn L. Lubrication of Poly(vinyl alcohol) Chain Orientation by Carbon Nano-Chips in Composite Tapes. J. Appl. Polym. Sci. 2013;127(4):2977-2982.

[30] Zhang Yiying, Song Kenan, Meng Jiangsha, Minus Marilyn L. Tailoring Polyacrylonitrile Interfacial Morphological Structure by Crystallization in the Presence of Single-Wall Carbon Nanotubes. ACS Applied Materials \& Interfaces 2013;5(3):807-814.

[31] Coleman Jonathan N., Cadek Martin, Ryan Kevin P., Fonseca Antonio, Nagy Janos B., Blau Werner J., et al. Reinforcement of polymers with carbon nanotubes. The role of an ordered polymer interfacial region. Experiment and modeling. Polymer 2006;47(26):8556-8561. [32] Song Kenan, Zhang Yiying, Meng Jiangsha, Green Emily, Tajaddod Navid, Li Heng, et al. Structural Polymer-Based Carbon Nanotube Composite Fibers: Understanding the ProcessingStructure-Performance Relationship. Materials 2013;6(6):2543-2577.

[33] Schadler Linda. Nanocomposites: Model interfaces. Natural Materials 2007;6(4):257-258.

[34] Schadler L. S., Brinson L. C., Sawyer W. G. Polymer nanocomposites: A small part of the story. Journal of the Minerals, Metals and Materials Society 2007;59(3):53-60.

[35] Sabina Prilutsky, Eyal Zussman, Yachin Cohen. The effect of embedded carbon nanotubes on the morphological evolution during the carbonization of poly(acrylonitrile) nanofibers. Nanotechnology 2008;19(16):165603.

[36] Liu Yaodong, Chae Han Gi, Kumar Satish. Gel-spun carbon nanotubes/polyacrylonitrile composite fibers. Part III: Effect of stabilization conditions on carbon fiber properties. Carbon 2011;49(13):4487-4496.

[37] Prilutsky Sabina, Zussman Eyal, Cohen Yachin. Carbonization of electrospun poly(acrylonitrile) nanofibers containing multiwalled carbon nanotubes observed by transmission electron microscope with in situ heating. Journal of Polymer Science Part B: Polymer Physics 2010;48(20):2121-2128. 
[38] Zhang Yiying, Minus Marilyn L. Characterization and Structural Analysis of SolutionGrown Polyacrylonitrile-co-Methacrylic Acid (PAN-co-MAA) Single Crystals. Macromolecules 2014;47(12):3987-3996.

[39] Ebewele Robert O. Polymer Science and Technology. CRC Press LLC: Boca Raton, Florida, 2000.

[40] Nowacki R., Monasse B., Piorkowska E., Galeski A., Haudin J. M. Spherulite nucleation in isotactic polypropylene based nanocomposites with montmorillonite under shear. Polymer 2004;45(14):4877-4892.

[41] Hwang Wook Ryol, Peters Gerrit W. M., Hulsen Martien A., Meijer Han E. H. Modeling of flow-induced crystallization of particle-filled polymers. Macromolecules 2006;39(24):8389-8398. [42] Xu J. Z., Chen C., Wang Y., Tang H., Li Z. M., Hsiao B. S. Graphene nanosheets and shear flow induced crystallization in isotactic polypropylene nanocomposites. Macromolecules 2011;44(8):2808-2818.

[43] Liu Yaodong, Kumar Satish. Polymer/Carbon Nanotube Nano Composite Fibers-A Review. ACS Applied Materials \& Interfaces 2014;6(9):6069-6087.

[44] Sano M., Sandberg M. O., Yoshimura S. Polymerization-induced epitaxy of polypeptides on graphite studied by scanning-tunneling-microscopy. Langmuir 1994;10(10):3815-3819.

[45] Li C. Y, Li L., Cai W., Kodjie S. L, Tenneti K. K. Nanohybrid shish-kebabs: Periodically functionalized carbon nanotubes. Advanced Materials 2005;17(9):1198-1202.

[46] Gupta A., Harrison I. R. New aspects in the oxidative stabilization of PAN-based carbon fibers. Carbon 1996;34(11):1427-1445.

[47] Gupta A., Harrison I. R. New aspects in the oxidative stabilization of PAN-based carbon fibers: II. Carbon 1997;35(6):809-818.

[48] Wang Zhonglin, Hui Chun. Electron Microscopy of Nanotubes. Kluwer Academic Publishers: Boston/Dordrecht/London, 2003.

[49] Lanticse-Diaz Leslie Joy, Tanabe Yasuhiro, Enami Takashi, Nakamura Kazumasa, Endo Morinobu, Yasuda Eiichi. The effect of nanotube alignment on stress graphitization of carbon/carbon nanotube composites. Carbon 2009;47(4):974-980.

[50] Zhou Zhou, Wang Xin, Faraji Shaghayegh, Bradford Philip D., Li Qingwen, Zhu Yuntian. Mechanical and electrical properties of aligned carbon nanotube/carbon matrix composites. Carbon 2014;75(0):307-313.

[51] Koganemaru Ai, Bin Yuezhen, Tohora Hidekazu, Okino Fujio, Komiyama Shingo, Zhu John, et al. Carbonization of oriented polyacrylonitrile and multiwalled carbon nanotube composite films. Asia-Pacific Journal of Chemical Engineering 2008;3(5):521-526.

[52] Maitra T., Sharma S., Srivastava A., Cho Y. K., Madou M., Sharma A. Improved graphitization and electrical conductivity of suspended carbon nanofibers derived from carbon nanotube/polyacrylonitrile composites by directed electrospinning. Carbon 2012;50(5):17531761.

[53] Meng Jiangsha, Zhang Yiying, Cranford Steven W., Minus Marilyn L. Nanotube Dispersion and Polymer Conformational Confinement in a Nanocomposite Fiber: A Joint Computational Experimental Study. The Journal of Physical Chemistry B 2014;118(31):9476-9485.

[54] De Fonton S., Oberlin A., Inagaki M. Characterization by electron microscopy of carbon phases (intermediate turbostratic phase and graphite) in hard carbons when heat-treated under pressure. Journal of Materials Science 1980;15(4):909-917.

[55] Chung D. D. L. Review Graphite. Journal of Materials Science 2002;37(8):1475-1489. 
[56] Ebbesen Thomas W. Carbon Nanotubes: Preparation and Properties. CRC Press, Inc.: Boca Raton, 1997.

[57] Li Hui, Yang ChuanZheng, Liu Fang. Novel method for determining stacking disorder degree in hexagonal graphite by X-ray diffraction. Sci. China Ser. B-Chem. 2009;52(2):174-180. [58] Beguin Francois, Frackowiak Elzbieta. Carbons for Electrochemical Energy Storage and Conversion Systems. Taylor and Francis Group, LLC: Boca Raton, 2010.

[59] Ho C. H., Chang C. P., Su W. P., Lin M. F. Precessing anisotropic Dirac cone and Landau subbands along a nodal spiral. New Journal of Physics 2013;15(5):053032.

[60] Pang Louis S. K., Saxby John D., Chatfield S. Peter. Thermogravimetric analysis of carbon nanotubes and nanoparticles. The Journal of Physical Chemistry 1993;97(27):6941-6942.

[61] Snead L. L., Burchell T. D. Thermal conductivity degradation of graphites due to nuetron irradiation at low temperature. Journal of Nuclear Materials 1995;224(3):222-229. 